\title{
Laplacians on infinite graphs: Dirichlet and Neumann boundary conditions
}

\author{
Sebastian Haeseler, Matthias Keller, Daniel Lenz, and Radosław Wojciechowski
}

\begin{abstract}
We study Laplacians associated to a graph and single out a class of such operators with special regularity properties. In the case of locally finite graphs, this class consists of all selfadjoint, non-negative restrictions of the standard formal Laplacian and we can characterize the Dirichlet and Neumann Laplacians as the largest and smallest Markovian restrictions of the standard formal Laplacian. In the case of general graphs, this class contains the Dirichlet and Neumann Laplacians and we describe how these may differ from each other, characterize when they agree, and study connections to essential selfadjointness and stochastic completeness.

Finally, we study basic common features of all Laplacians associated to a graph. In particular, we characterize when the associated semigroup is positivity improving and present some basic estimates on its long term behavior. We also discuss some situations in which the Laplacian associated to a graph is unique and, in this context, characterize its boundedness.
\end{abstract}

Mathematics Subject Classification (2010). Primary 47B25; Secondary 05 C63.

Keywords. Laplacian on graphs, Dirichlet boundary conditions, Neumann boundary conditions, Markovian extension.

\section{Contents}

1 Introduction . . . . . . . . . . . . . . . . . . . . 398

2 Framework and basic results . . . . . . . . . . . . . 400

3 Laplacians associated to a graph . . . . . . . . . . . . . . . . 404

4 The forms $Q^{(D)}$ and $Q^{(N)} \ldots \ldots \ldots \ldots \ldots . \ldots . \ldots 40 . \ldots \ldots$

5 Characterizing Neumann and Dirichlet Laplacians . . . . . . . . . . . 411

6 The equation $(\tilde{L}+1) u=0 \ldots \ldots \ldots \ldots \ldots$. . . . . . . . 417

7 Maximum principle and characterization of positivity improvement . . . . 421

8 An analogue to a theorem of $\mathrm{Li} \ldots \ldots \ldots . \ldots 422$

9 Uniqueness of selfadjoint restrictions of $\tilde{L} \ldots \ldots \ldots$. . . . . . . . . 424

A. A (counter)example . . . . . . . . . . . . . . . . 426

B. Dirichlet forms on real and complex Hilbert spaces . . . . . . . . . 427

References . . . . . . . . . . . . . . . . . . . . . . . . . 429 


\section{Introduction}

Laplacians on graphs have been studied for a long time; see, e.g., the monographs [3], [5], and references therein. Much of the research has been devoted to finite graphs and bounded Laplacians.

After sporadic earlier investigations, notably by Dodziuk [9] and Mohar [33], certain properties related to unboundedness of the associated Laplacians on infinite graphs have become a focus of attention in recent years. This concerns, in particular, essential selfadjointness [6], [10], [17], [18], [23], [24], [26], [32], [37], [38], and [39], stochastic (in)completeness [10], [12], [19], [22], [26], [27], [38], [39], [40], and [41], and suitable isoperimetric inequalities [4], [10], [16], [25], [27], [29], [39], and [40]; see references in the cited works for further literature as well.

It turns out that all of these works deal with what could be called the "Dirichlet Laplacian" on a graph. In the essentially selfadjoint case, of course, this is the only Laplacian. In general, however, further selfadjoint Laplacians exist. In particular, there exists a "Neumann Laplacian". It is not clear when the two Laplacians agree and which properties they share (if they do not agree). This is the starting point of this paper. More generally, our aim is to investigate the following three related questions:

(Q-1) Which operators can be considered to be Laplacians associated to a graph?

(Q-2) How are these operators related and what are the differences between them?

(Q-3) What are the basic properties common to all of them?

We now provide a general overview of the paper and our results on these questions. For precise statements and definitions of the quantities involved we refer to later sections.

In Section 2, we give an exposition of basic notation and concepts. In particular, we introduce graphs, the standard formal Laplacian associated to a graph, and the forms $Q^{(D)}$ and $Q^{(N)}$ giving the Dirichlet and Neumann Laplacians, respectively. We also prove a result showing that the "weak domain" of definition of the formal Laplacian actually agrees with its domain (Theorem 2.2). This result is important for our further considerations and may also be of independent interest.

As for (Q-1), which is studied in Section 3, we note that any graph comes with both a standard formal operator $\widetilde{L}$ and a closed form $Q^{(D)}$. In some sense, $\tilde{L}$ is the "maximal" Laplacian associated to the graph and $Q^{(D)}$ is the "largest" closed form associated to $\widetilde{L}$. This leads us to single out Laplacians and forms associated to a graph which satisfy a regularity-type condition, called $(\mathcal{C})$, implying that the form lies between $Q^{(D)}$ and $\widetilde{L}$. A precise concept is given in Definition 3.1.

In the case of locally finite graphs, the corresponding Laplacians turn out to be exactly the selfadjoint restrictions of $\widetilde{L}$ which are bounded below (Theorem 3.10 ). In the case of general graphs, we do not have an explicit description of all Laplacians satisfying $(\mathcal{C})$ in terms of $\tilde{L}$. However, we can show that the Dirichlet operator and the Neumann operator (and all operators between them in the sense of forms) satisfy 
this condition (Proposition 3.8). In this sense, our framework seems to be sufficient to address questions (Q-2) and (Q-3) and, in particular, to study the Dirichlet and Neumann Laplacians.

As for (Q-2), our framework allows us to obtain, in an easy way, a general description of how a form satisfying $(\mathcal{C})$ can be seen as an extension of $Q^{(D)}$. This is given in Theorem 3.6 of Section 3. This theorem can be seen as a form-type analogue of some basic results in von Neumann extension theory. On a technical level, the main topic is the description of 1-harmonic functions $u$ in the domain of the form $Q$ associated to the graph, i.e. $u$ with

$$
(\widetilde{L}+1) u=0
$$

belonging to the space $D(Q)$.

In Sections 4 and 5, we then have a closer look at (Q-2) for Dirichlet and Neumann Laplacians. In Section 4, we describe the "difference" between Dirichlet and Neumann Laplacians if they do not agree (Theorem 4.2) and give a characterization of when the Dirichlet and Neumann Laplacians agree (Corollary 4.3). We also discuss how our results are related to recent work of Colin de Verdière, Torki-Hamza and Truc; see [6], and [37]. In fact, while somewhat different in spirit, our description of the difference between Neumann and Dirichlet Laplacians in Theorem 4.2 is certainly inspired by [6].

We then turn to characterizing Dirichlet and Neumann Laplacians in the framework of Laplacians associated to a graph in Section 5. Our approach gives immediately that the Dirichlet Laplacian is, in a precise sense, the largest Laplacian associated to a graph. The main thrust of Section 5 is to show that the Neumann Laplacian is the smallest Laplacian associated to a graph within the class of Markovian operators (i.e. operators associated to a Dirichlet form). For our results to work, we have to make the additional assumption of local finiteness of the graph. For locally finite graphs, Theorem 5.2 then gives that, among the Markovian restrictions of $\widetilde{L}$, the Dirichlet Laplacian is the biggest and the Neumann Laplacian is the smallest. While similar results are known for the usual Laplacians on subsets of Euclidean space [15], we are not aware of any earlier result of this type for graphs.

It is remarkable that the agreement of $Q^{(D)}$ and $Q^{(N)}$ is equivalent to the triviality of solutions to $(\widetilde{L}+1) u=0$ in $D\left(Q^{(N)}\right)$, as the solvability of this equation in other spaces is known to be related to stochastic completeness and to essential selfadjointness. In this way, essential selfadjointness, stochastic completeness, and uniqueness of the operator are related. Details are discussed in Section 6. In particular, by examples we show that, apart from the "obvious" implications, no implications between these concepts hold in general. More specifically, we show that stochastic completeness and essential selfadjointness are not related in general.

We finally turn to question (Q-3) and discuss basics of a theory valid for both Neumann and Dirichlet Laplacians (and many others) in Sections 7 and 8. There, we are mostly concerned with the semigroup associated to these operators. 
First, we present a maximum principle for solutions of $(\tilde{L}+1) u=0$ and use it to characterize when the semigroup is positivity improving in Section 7. This generalizes the corresponding considerations for the Dirichlet Laplacian in [26]; see [38], [39], and [8] for earlier treatment of special Dirichlet Laplacians as well.

We then discuss an analogue to a result of $\mathrm{Li}$ on Laplacians on manifolds in our context in Section 8. This result has already been obtained recently in a rather general context [28]. Here, we present a different proof which is adapted to the graph case.

In Section 9, we conclude the paper with a study of situations in which there is only one selfadjoint restriction of $\widetilde{L}$. This complements and completes the considerations of the earlier sections which deal with the differences and common features of all selfadjoint restrictions.

In some sense, this paper can be seen as a complement to [26]. There, basic features of the Dirichlet Laplacian were discussed. Here, we focus on the general case.

Acknowledgements. Part of this work was done while the authors were visiting the workshop Analysis on Graphs and its Applications Follow-up Meeting at the Isaac Newton Institute. The authors would like to thank the organizers for the invitation and gratefully acknowledge the stimulating atmosphere of the workshop. In this context D. Lenz, S. Haeseler, and M. Keller also gratefully acknowledge partial support by the German Science Foundation (DFG). R. Wojciechowski acknowledges the financial support of FCT grant SFRH/BPD/45419/2008 and FCT project PTDC/MAT/101007/2008.

\section{Framework and basic results}

Throughout the paper, let $V$ be a finite or countably infinite set and $m$ a measure on $V$ with full support (i.e. $m$ is a map on $V$ taking values in $(0, \infty))$. We then call $(V, m)$ a discrete measure space. The set of all function from $V$ to $\mathbb{C}$ is denoted by $C(V)$.

We will introduce operators on $\ell^{2}(V, m)$ using Dirichlet forms. To do so, we first briefly recall a few standard facts on forms; see, e.g., [8] and [15]. Some of the standard literature on Dirichlet forms only deals with real Hilbert spaces. However, this can easily be extended to complex Hilbert spaces. Some details are discussed in Appendix B. A form $Q$ on a (complex) Hilbert space with domain of definition given by a dense subspace $D(Q)$ is a sesquilinear map $Q: D(Q) \times D(Q) \rightarrow \mathbb{C}$. The form $Q$ is called non-negative if $Q(u, u) \geq 0$ for all $u \in D(Q)$ and symmetric if $Q(u, v)=\overline{Q(v, u)}$ for all $u, v \in D(Q)$. A non-negative symmetric form $Q$ is called closed if $D(Q)$ with the inner product

$$
\langle u, v\rangle_{Q} \stackrel{\text { def }}{=} Q(u, v)+\langle u, v\rangle
$$

is complete, i.e. a Hilbert space. To each such form there exists a unique selfadjoint 
operator $L$ with

$$
D(Q)=\text { Domain of definition of } L^{1 / 2}
$$

and

$$
Q(u, v)=\left\langle L^{1 / 2} u, L^{1 / 2} v\right\rangle .
$$

A map $C: \mathbb{C} \rightarrow \mathbb{C}$ with $C(0)=0$ and $|C(x)-C(y)| \leq|x-y|$ is called a normal contraction. A closed form $Q$ on a Hilbert space of square integrable functions is called a Dirichlet form if

$$
Q(C u, C u) \leq Q(u, u)
$$

for all $u \in D(Q)$ and all normal contractions $C$. The relevance of Dirichlet forms comes from the fact that the associated semigroups $\left(e^{-t L}\right)_{t \geq 0}$ and resolvents $\alpha(L+$ $\alpha)^{-1}, \alpha>0$, are positivity preserving, i.e. map non-negative functions to non-negative functions and provide contractions on the space of bounded functions; see, e.g., [1] and [7].

After this summary on forms, we now come to a discussion of graphs over $(V, m)$ and the associated operators. To a large extent we follow [26] and [21] to which we refer for further details and proofs not given below. (Note that our notation deviates from the notations of [26] and [21] - which are only concerned with the Dirichlet Laplacian - in the following way: We denote by $Q^{(N)}$ the form denoted by $Q^{\text {max }}$ in [26] and by $Q^{(D)}$ the form denoted by $Q$ in [26].)

By a symmetric weighted graph over $V$ we mean a pair $(b, c)$ consisting of a map $c: V \rightarrow[0, \infty)$ and a map $b: V \times V \rightarrow[0, \infty)$ satisfying the following properties:

- $b(x, x)=0$ for all $x \in V$;

- $b(x, y)=b(y, x)$ for all $x, y \in V$;

- $\sum_{y \in V} b(x, y)<\infty$ for all $x \in V$.

Then, $x, y \in V$ with $b(x, y)>0$ are called neighbors and thought to be connected by an edge with weight $b(x, y)$. More generally, $x, y \in V$ are called connected by the path $\left(x_{0}, x_{1}, \ldots, x_{n+1}\right)$ if $x_{0}, x_{1}, \ldots, x_{n+1} \in V$ satisfy $b\left(x_{i}, x_{i+1}\right)>0$, $i=0, \ldots, n$, with $x_{0}=x$ and $x_{n+1}=y$. A connected component of the graph is a maximal subset of $V$ such that all elements in this set are connected. If $V$ has only one connected component, i.e., if any two $x, y \in V$ are connected, then $(b, c)$ is called connected. Symmetric weighted graphs over $(V, m)$ are also known as symmetric Markov chains over $(V, m)$.

We are now going to associate forms and operators to each graph $(b, c)$ over $(V, m)$. These forms and operators will, of course, depend on the choice of $(b, c, m)$. We will mostly omit this dependence on $(b, c, m)$ in our notation and only add the corresponding subscripts when necessary to avoid confusion.

To the graph $(b, c)$ over $(V, m)$ we associate the form $Q^{(N)}$ on the Hilbert space $\ell^{2}(V, m)$ with domain of definition $D\left(Q^{(N)}\right)$ given by the subspace

$$
D\left(Q^{(N)}\right) \stackrel{\text { def }}{=}\left\{u \in \ell^{2}(V, m): \frac{1}{2} \sum_{x, y \in V} b(x, y)|u(x)-u(y)|^{2}+\sum_{x \in V} c(x)|u(x)|^{2}<\infty\right\}
$$


and the map

$$
Q^{(N)}: D\left(Q^{(N)}\right) \times D\left(Q^{(N)}\right) \longrightarrow \mathbb{C}
$$

by

$$
Q^{(N)}(u, v) \stackrel{\text { def }}{=} \frac{1}{2} \sum_{x, y \in V} b(x, y)(u(x)-u(y)) \overline{(v(x)-v(y))}+\sum_{x \in V} c(x) u(x) \overline{v(x)} .
$$

Then, $Q^{(N)}$ is symmetric, non-negative and closed. The associated operator will be denoted by $L^{(N)}$. We can think of $L^{(N)}$ as a Laplacian with Neumann-type boundary conditions.

We will be concerned not only with $Q^{(N)}$ but with further forms as well. In this context, we use the notation $Q_{1} \subseteq Q_{2}$ to mean that

$$
D\left(Q_{1}\right) \subseteq D\left(Q_{2}\right) \quad \text { and } \quad Q_{1}(u, v)=Q_{2}(u, v)
$$

for all $u, v \in D\left(Q_{1}\right)$. Similarly, for non-negative forms $Q_{1}$ and $Q_{2}$, we use the notation $Q_{1} \leq Q_{2}$ to mean that

$$
D\left(Q_{2}\right) \subseteq D\left(Q_{1}\right) \quad \text { and } \quad Q_{1}(u, u) \leq Q_{2}(u, u)
$$

for all $u \in D\left(Q_{2}\right)$.

Obviously, the set $C_{c}(V)$ of functions from $C(V)$ with finite support belongs to $D\left(Q^{(N)}\right)$. Thus, we can restrict $Q^{(N)}$ to this set to obtain the form $Q^{\text {comp }}$

$$
Q^{\text {comp }}: C_{c}(V) \times C_{c}(V) \longrightarrow \mathbb{C}, \quad Q^{\text {comp }}(u, v) \stackrel{\text { def }}{=} Q^{(N)}(u, v) .
$$

The form $Q^{\text {comp }}$ is not closed but possesses a unique smallest closed extension called the closure and denoted by $Q^{(D)}$. The associated selfadjoint operator is denoted by $L^{(D)}$. We can think of $L^{(D)}$ as a Laplacian with Dirichlet-type boundary conditions.

Note that $Q^{(N)}$ and $Q^{(D)}$ are Dirichlet forms. This is rather straightforward to show for $Q^{(N)}$ and follows for $Q^{(D)}$ by general principles. By construction, the form $Q^{(D)}$ is a regular Dirichlet form, viz, $C_{c}(V)$ is dense in the form domain with respect to the form norm induced by $\langle\cdot, \cdot\rangle_{Q^{(D)}}$. In fact, all regular Dirichlet forms on $(V, m)$ are of the form $Q^{(D)}=Q_{(b, c)}^{(D)}$ for suitable graphs $(b, c)$; see, e.g., [26].

While the domains of definition of $L^{(D)}$ and $L^{(N)}$ can, in general, not be described explicitly, the action of these operators is easily described. To do so, we introduce the standard formal Laplacian $\widetilde{L}$ associated to the graph $(b, c)$ over $(V, m)$. This operator will be of fundamental importance in all of our considerations. It is defined on the space

$$
\widetilde{F} \stackrel{\text { def }}{=}\left\{u \in C(V): \sum_{y \in V}|b(x, y) u(y)|<\infty \text { for all } x \in V\right\}
$$


by

$$
\widetilde{L} u(x) \stackrel{\text { def }}{=} \frac{1}{m(x)} \sum_{y \in V} b(x, y)(u(x)-u(y))+\frac{c(x)}{m(x)} u(x) .
$$

Note that, for each $x \in V$, the sum exists by the assumption that $u$ belongs to $\widetilde{F}$.

It turns out that $\widetilde{L}$ has a certain regularity property, viz, functions which are weakly in its domain are actually in its domain. The crucial identity connecting $\widetilde{L}$ and the forms we have in mind is then given by a certain integration by parts. This is discussed next. We start by introducing the functions which are weakly in the domain of $\widetilde{L}$; see [14] as well.

Definition 2.1. Let $(V, m)$ be a discrete measure space and $(b, c)$ a graph over $(V, m)$. Then, $\widetilde{F}^{*}$, the weak domain of the formal Laplacian, is defined by

$$
\tilde{F}^{*} \stackrel{\text { def }}{=}\left\{u \in C(V): \sum_{x \in V}|u(x) \tilde{L} v(x)| m(x)<\infty \text { for all } v \in C_{c}(V)\right\} .
$$

Here, comes the first part of the necessary "integration by parts" as shown in [21] (see [26] for related results as well). For $u \in \widetilde{F}$ and $v \in C_{c}(V)$, the sum

$$
\widetilde{Q}(u, v) \stackrel{\text { def }}{=} \frac{1}{2} \sum_{x, y \in V} b(x, y)(u(x)-u(y))(\overline{v(x)-v(y)})+\sum_{x \in V} c(x) u(x) \overline{v(x)}
$$

converges absolutely and the equality

$$
\widetilde{Q}(u, v)=\sum_{x \in V} \tilde{L} u(x) \overline{v(x)} m(x)=\sum_{x \in V} u(x) \overline{\widetilde{L} v(x)} m(x)
$$

holds (where all sums are converging absolutely).

After these preparations we can now state a regularity property of $\widetilde{L}$.

Theorem 2.2. Let $(V, m)$ be a discrete measure space and $(b, c)$ a graph over $(V, m)$. Then, $\widetilde{F}=\widetilde{F}^{*}$.

Proof. The inclusion $\widetilde{F} \subseteq \widetilde{F}^{*}$ follows from (1). It remains to show the other inclusion $\widetilde{F}^{*} \subseteq \widetilde{F}$. Let $u \in \widetilde{F}^{*}$ be given. We have to show the absolute convergence of $\sum_{z \in V} b(x, z) u(z)$ for any $x \in V$. Let $\delta_{x}$ be the characteristic function of $\{x\}$. For each $z \in V$, we set

$$
B_{z} \stackrel{\text { def }}{=} \sum_{y \in V} b(z, y)+c(z) .
$$

Then, a direct calculation shows that

$$
\widetilde{L} \delta_{x}(z)=\frac{1}{m(z)}\left(B_{z} \delta_{x}(z)-b(x, z)\right) .
$$

As $\delta_{x}$ belongs to $C_{c}(V)$, the absolute convergence of $\sum u(z) \tilde{L} \delta_{x}(z) m(z)$ for each $x \in V$ follows by the assumption on $u$. Now, $(*)$ easily gives the statement. 
Remark. The previous theorem seems particularly remarkable to us as it does not seem to have a direct counterpart in the case of the usual Laplace-Beltrami $\Delta_{M}$ on a Riemannian manifold $M$. Certainly, the existence of $\langle u, \Delta v\rangle$ for all $v \in C_{c}^{\infty}(M)$ does not imply any differentiability properties of $u$ (as it will hold, in particular, for any measurable bounded function with compact support).

As a consequence of the previous theorem we obtain that weak generalized eigenfunctions are generalized eigenfunctions.

Corollary 2.3. Let $(V, m)$ be a discrete measure space, $(b, c)$ a graph over $(V, m)$, $u \in C(V)$ and $\lambda \in \mathbb{R}$. Then, the following assertions are equivalent:

(i) the function $u$ belongs to $\widetilde{F}$ and $(\widetilde{L}-\lambda) u=0$, i.e. $u$ is a generalized eigenfunction of $\widetilde{L}$ to the eigenvalue $\lambda$.

(ii) the function $u$ belongs to $\widetilde{F}^{*}$ and $\sum_{x \in V} u(x) \overline{(\widetilde{L}-\lambda) v(x)} m(x)=0$ for all $v \in C_{c}(V)$, i.e. $u$ is a weak generalized eigenfunction of $\widetilde{L}$.

\section{Laplacians associated to a graph}

In this section, we introduce a special class of operators and forms associated to a graph. As will become clear in the paper, these forms and operators can be considered as particularly regular Laplacians on a graph. In this section, we develop some basics of their theory. In particular, Theorem 3.6 gives a form-type analogue of what might be seen as a basic ingredient of von Neumann extension theory for symmetric operators. Moreover, we show that all the "usual" Laplacians fall into our framework. More precisely, we show in Theorem 3.10 that, in the locally finite case (and even a bit more generally), our class consists of the non-negative selfadjoint restrictions of $\widetilde{L}$. In the case of general graphs, we show that the Dirichlet and Neumann operators (and all operators between them in the sense of forms) belong to the class.

Whenever we are given a graph with an associated standard formal Laplacian $\widetilde{L}$ we call a selfadjoint restriction of $\widetilde{L}$ a Laplacian associated to the graph. If this restriction is bounded below, we call the induced form a form associated to the graph. We are going to single out a special class of operators associated to a graph and study some of their properties. We start with the definition of the class.

Definition 3.1 (Forms satisfying $(\mathcal{C})$ ). Let $(V, m)$ be a discrete measure space, $(b, c)$ a graph over $(V, m)$ and $\widetilde{L}=\widetilde{L}_{(b, c, m)}$. A symmetric form $Q$ on $\ell^{2}(V, m)$ with domain $D$ is said to satisfy condition (e) with respect to $(b, c)$ if

(C0) $Q$ is non-negative and closed,

(C1) $C_{c}(V) \subseteq D$, 
(C2) For any $u \in D$ and any $v \in C_{c}(V)$ the sum $\sum_{x \in V} u(x) \tilde{L} v(x) m(x)$ converges absolutely and the equality

$$
Q(u, v)=\sum_{x \in V} u(x) \overline{\widetilde{L} v(x)} m(x)
$$

holds.

The selfadjoint operator $L$ induced by the form is then also said to satisfy $(\mathcal{C})$.

Remark. The requirement in $(\mathrm{C} 0)$ that $Q$ is non-negative could be replaced by the assumption that $Q$ is bounded below (with appropriate changes). We assume that $Q \geq 0$ in order to simplify the notation later and not have to worry about some constants.

Theorem 2.2 combined with (1) allows us to restate condition (C2) as follows.

Corollary 3.2. Let $(V, m)$ be a discrete measure space and $(b, c)$ a graph over $(V, m)$. Let the form $Q$ with domain $D$ satisfy $(\mathcal{C})$ with respect to $(b, c)$. Then, $D \subseteq \widetilde{F}$ and

$$
Q(u, v)=\sum_{x \in V} \tilde{L} u(x) \overline{v(x)} m(x)
$$

holds for all $u \in D$ and $v \in C_{c}(V)$.

The next proposition gathers some basic properties of forms and operators satisfying $(\mathcal{C})$ (and gives, in particular, that they are associated to a graph). Recall from Section 2 the definition of $\langle\cdot, \cdot\rangle_{Q}$ via

$$
\langle u, v\rangle_{Q}=Q(u, v)+\langle u, v\rangle .
$$

Proposition 3.3. Let $(V, m)$ be a discrete measure space, $(b, c)$ a graph over $(V, m)$ and $Q$ a form with domain $D$ satisfying $(\mathcal{C})$ with respect to $(b, c)$. Then, the following properties hold:

(a) $D\left(Q^{(D)}\right)$ is a closed subspace of the Hilbert space $\left(D,\langle\cdot, \cdot\rangle_{Q}\right)$ and $Q^{(D)}$ and $Q$ agree on $D\left(Q^{(D)}\right)$;

(b) the selfadjoint operator $L$ associated to $Q$ is a restriction of $\tilde{L}$ and is nonnegative.

Proof. (a) Note that $Q$ agrees with $Q^{(D)}$ on $C_{c}(V)$ by the assumptions on $Q$. Thus, the closure of $C_{c}(V)$ with respect to $\langle\cdot, \cdot\rangle_{Q}$ is exactly $D\left(Q^{(D)}\right)$ and $D\left(Q^{(D)}\right)$ is a closed subspace of the Hilbert space $\left(D,\langle\cdot, \cdot\rangle_{Q}\right)$.

(b) This is immediate from Corollary 3.2. 
A direct consequence of the previous proposition is the following maximality property of $Q^{(D)}$.

Corollary 3.4. Let $(V, m)$ be a discrete measure space and $(b, c)$ a graph over $(V, m)$. Then, $Q^{(D)} \subseteq Q$ and, in particular, $Q \leq Q^{(D)}$ holds for any form $Q$ satisfying ( $\left.\mathcal{C}\right)$ with respect to $(b, c)$.

In order to state our main abstract result on the description of forms satisfying $(\mathcal{C})$ we need one further piece of notation.

Definition 3.5 (Harmonic function). Let $(V, m)$ be a discrete measure space and $(b, c)$ a graph over $(V, m)$. For a form $Q$ with domain $D$ satisfying $(\mathcal{C})$ with respect to $(b, c)$, the space of 1-Q-harmonic functions $\mathscr{H}^{(Q)}$ is defined by

$$
\mathscr{H}^{(Q)} \stackrel{\text { def }}{=}\{u \in D:(\tilde{L}+1) u=0\} .
$$

Remark. By Corollary 2.3, the space $\mathscr{H}^{(Q)}$ could also be defined via "weak solutions", i.e.

$$
\mathscr{H}^{(Q)}=\left\{u \in D: \sum_{x \in V} u(x) \overline{(\widetilde{L}+1) v(x)} m(x)=0 \text { for all } v \in C_{c}(V)\right\} .
$$

Here is the main result of this section.

Theorem 3.6. Let $(V, m)$ be a discrete measure space and $(b, c)$ a graph over $(V, m)$. Let the form $Q$ with domain $D$ satisfy $(\mathcal{C})$ with respect to $(b, c)$. Then, for $u \in D$, the following assertions are equivalent:

(i) $(\tilde{L}+1) u=0$;

(ii) $u$ is orthogonal to $D\left(Q^{(D)}\right)$ with respect to the inner product $\langle\cdot, \cdot\rangle_{Q}$.

Therefore, the Hilbert space $\left(D,\langle\cdot, \cdot\rangle_{Q}\right)$ can be decomposed as an orthogonal sum

$$
D=D\left(Q^{(D)}\right) \oplus \mathscr{H}^{(Q)}
$$

Proof. It suffices to show the equivalence of (i) and (ii). The remaining statement is then immediate. Now, obviously, $(\tilde{L}+1) u=0$ is equivalent to

$$
\sum_{x \in V}(\tilde{L}+1) u(x) \overline{v(x)} m(x)=0
$$

for any $v \in C_{c}(V)$. By Corollary 3.2, this is equivalent to

$$
0=Q(u, v)+\langle u, v\rangle=\langle u, v\rangle_{Q}
$$

for all $v \in C_{c}(V)$. As $D\left(Q^{(D)}\right)$ is the closure of $C_{c}(V)$ in $D$ with respect to $\langle\cdot, \cdot\rangle_{Q}$, we obtain the desired equivalence. 
Corollary 3.7. Let $(V, m)$ be a discrete measure space and $(b, c)$ a graph over $(V, m)$. Let the form $Q$ with domain $D$ satisfy $(\mathcal{C})$ with respect to $(b, c)$. Define $B \stackrel{\text { def }}{=} \mathscr{B}(Q) \stackrel{\text { def }}{=}$ $D / D\left(Q^{(D)}\right)$. Then, for each $v \in \mathscr{B}$ there exists a unique $w=w_{v} \in D$ with

- $(\widetilde{L}+1) w=0$

- $[w]=v$

and the map

$$
\mathscr{B} \longrightarrow \mathscr{H}^{(Q)}, \quad v \longmapsto w_{v},
$$

is a bijection and even a unitary (if both vector spaces are equipped with the induced Hilbert space structure).

Remark. One can think of $\mathcal{B}(Q)$ as a general type of boundary value of the elements of $D$. Accordingly, the corollary gives the existence and uniqueness of a solution to a boundary value problem.

After this discussion of general features of the class of operators and forms satisfying $(\mathcal{C})$, we now discuss important examples of such operators. First, we show that the forms $Q^{(N)}$ and $Q^{(D)}$, and all closed forms between them, belong to this class.

Proposition 3.8. Let $(V, m)$ be a discrete measure space and $(b, c)$ a graph over $(V, m)$. Then, any closed form $Q$ with $Q^{(D)} \subseteq Q \subseteq Q^{(N)}$ satisfies $(\mathcal{C})$ with respect to $(b, c)$. In particular, the selfadjoint operator $L$ associated to such a form $Q$ is a restriction of $\widetilde{L}$.

Proof. It suffices to show that $Q^{(N)}$ satisfies ( $\left.\mathcal{C}\right)$. By (1), it suffices to show that $D\left(Q^{(N)}\right) \subseteq \widetilde{F}$. To see this, we let $w \in D\left(Q^{(N)}\right)$ and $B_{x} \stackrel{\text { def }}{=} \sum_{y} b(x, y)<\infty$ for each $x \in V$ and calculate

$$
\begin{aligned}
\sum_{y \in V} b(x, y)|w(y)| & \leq \sum_{y \in V} b(x, y)|w(x)-w(y)|+\sum_{y \in V} b(x, y)|w(x)| \\
& \leq\left(\sum_{y \in V} b(x, y)\right)^{\frac{1}{2}}\left(\sum_{y \in V} b(x, y)|w(x)-w(y)|^{2}\right)^{\frac{1}{2}}+B_{x}|w(x)| \\
& \leq B_{x}^{1 / 2} Q^{(N)^{1 / 2}}(w, w)+B_{x}|w(x)| .
\end{aligned}
$$

This gives the desired finiteness.

We now turn to a situation in which we can explicitly describe all Laplacians satisfying $(\mathcal{C})$.

Recall from [26] that for graphs $(b, c)$ over $(V, m)$ the following two conditions are equivalent:

- $\widetilde{L} C_{c}(V) \subseteq \ell^{2}(V, m)$;

- $b(x, \cdot) / m(\cdot) \in \ell^{2}(V, m)$ for all $x \in V$. 
A graph satisfying one (and then both) of these conditions will be said to satisfy the finiteness condition (FC).

For such graphs, we can define the minimal operator $L_{c}$ to be the restriction of $\widetilde{L}$ to $C_{c}(V)$ and the maximal operator $L_{M}$ to be restriction of $\widetilde{L}$ to

$$
D\left(L_{M}\right) \stackrel{\text { def }}{=}\left\{u \in \ell^{2}(V, m): \widetilde{L} u \in \ell^{2}(V, m)\right\} .
$$

In this situation, the following consequence of (1) holds (see [26] for details).

Proposition 3.9. Let $(V, m)$ be a discrete measure space and $(b, c)$ a graph over $(V, m)$ satisfying (FC). Then, $L_{M}$ is the adjoint of $L_{c}$ and, in particular, the set of selfadjoint restrictions of $\widetilde{L}$ is exactly the set of selfadjoint extensions of $L_{c}$.

A special instance of graphs satisfying (FC) are locally finite graphs. Here, a graph $(b, c)$ over $(V, m)$ is called locally finite if, for any $x \in V$, the set

$$
\{y \in V: b(x, y)>0\}
$$

is finite. In this case, the previous proposition can be strengthened and it follows that $\widetilde{F}$ is equal to $C(V), \widetilde{L}$ maps $C_{c}(V)$ into itself and, by (1), $\widetilde{L}$ can easily be seen to be the adjoint of the restriction $L_{c}$ with respect to the dual pairing $C(V) \times C_{c}(V) \rightarrow \mathbb{C}$, $(u, v) \mapsto \sum_{x} u(x) \overline{v(x)} m(x)$.

Our characterization of all Laplacians satisfying $(\mathcal{C})$ on graphs for which (FC) holds now follows.

Theorem 3.10. Let $(V, m)$ be a discrete measure space and $(b, c)$ a graph over $(V, m)$ satisfying (FC). Let $L$ be a non-negative selfadjoint operator on $\ell^{2}(V, m)$. Then, the following assertions are equivalent:

(i) L and its associated form $Q$ satisfy ( $\mathcal{C})$;

(ii) $L$ is a restriction of $\tilde{L}$.

Proof. The implication (i) $\Longrightarrow$ (ii) follows from Proposition 3.3 (and does not require (FC)). It remains to show the implication (ii) $\Longrightarrow$ (i). This is a simple consequence of Proposition 3.9 and (1).

\section{The forms $Q^{(D)}$ and $Q^{(N)}$}

In this section we study how $Q^{(D)}$ and $Q^{(N)}$ differ from each other. The difference will turn out to be essentially given by solutions of

$$
(\tilde{L}+1) u=0
$$

belonging to $D\left(Q^{(N)}\right)$. This will allow us to abstractly characterize when $Q^{(D)}$ and $Q^{(N)}$ agree. We then turn to a more geometric description of this difference suggested by recent results of [37] and [6]. 
Lemma 4.1. Let $(V, m)$ be a discrete measure space and $(b, c)$ a graph over $(V, m)$. If $Q^{(N)} \neq Q^{(D)}$, then there exists a non-trivial, non-negative solution to $(\tilde{L}+1) u=0$ in $D\left(Q^{(N)}\right) \cap \ell^{\infty}(V)$.

Proof. By $Q^{(N)} \neq Q^{(D)}$ we must have $L^{(N)} \neq L^{(D)}$ and then $\left(L^{(N)}+1\right)^{-1} \neq$ $\left(L^{(D)}+1\right)^{-1}$. As the vectors $\delta_{x}, x \in V$, are total in $\ell^{2}(V, m)$, there then exists an $x \in V$ such that

$$
u \stackrel{\text { def }}{=}\left(\left(L^{(N)}+1\right)^{-1}-\left(L^{(D)}+1\right)^{-1}\right) \delta_{x} \neq 0,
$$

where $\delta_{x}$ is the function in $\ell^{2}(V, m)$ which vanishes everywhere except at $x$ where it is 1 . As $Q^{(N)}$ and $Q^{(D)}$ are Dirichlet forms, both resolvents are contractions on $\ell^{\infty}(V)$ and the boundedness of $u$ follows. Thus, $u$ belongs to $\ell^{2}(V, m) \cap \ell^{\infty}(V)$. Moreover, $u$ belongs to $D\left(Q^{(N)}\right)$ as $\left(L^{(N)}+1\right)^{-1}$ maps into $D\left(L^{(N)}\right) \subseteq D\left(Q^{(N)}\right)$ and $\left(L^{(D)}+1\right)^{-1}$ maps into $D\left(L^{(D)}\right) \subseteq D\left(Q^{(D)}\right) \subseteq D\left(Q^{(N)}\right)$. As both $L^{(N)}$ and $L^{(D)}$ are restrictions of $\tilde{L}$, we obtain that $u$ solves

$$
(\tilde{L}+1) u=0 .
$$

Non-negativity of $u$ follows as $\left(L^{(D)}+1\right)^{-1} \delta_{x}$ is the smallest non-negative solution of $(\tilde{L}+1) v=\delta_{x}$ by Theorem 11 of [26].

By Proposition 3.8, the form $Q^{(N)}$ satisfies (C). Thus, we can now specialize Corollary 3.7 to obtain the following theorem on solving $(\widetilde{L}+1) u=0$ in $D\left(Q^{(N)}\right)$.

Theorem 4.2. Let $(V, m)$ be a discrete measure space, $(b, c)$ a graph over $(V, m)$ and $\mathscr{B}^{(N)} \stackrel{\text { def }}{=} D\left(Q^{(N)}\right) / D\left(Q^{(D)}\right)$. Then, for each $v \in \mathscr{B}^{(N)}$ there exists a unique $w=w_{v} \in D\left(Q^{(N)}\right)$ with

- $(\tilde{L}+1) w=0$,

- $[w]=v$.

As a corollary we obtain the following characterization of $Q^{(N)} \neq Q^{(D)}$.

Corollary 4.3 (Characterization of $\left.Q^{(N)} \neq Q^{(D)}\right)$. Let $(V, m)$ be a discrete measure space, $(b, c)$ a graph over $(V, m)$ and $\mathcal{B}^{(N)}=D\left(Q^{(N)}\right) / D\left(Q^{(D)}\right)$. Then, the following assertions are equivalent:

(i) $\mathcal{B}^{(N)} \neq\{0\}$, i.e. $Q^{(N)} \neq Q^{(D)}$;

(ii) there exists a non-trivial solution of $(\tilde{L}+1) u=0$ in $D\left(Q^{(N)}\right)$;

(iii) there exists a non-trivial solution of $(\tilde{L}+1) u=0$ in $D\left(Q^{(N)}\right) \cap \ell^{\infty}(V)$.

Proof. The implication (iii) $\Longrightarrow$ (ii) is clear. The implication (ii) $\Longrightarrow$ (i) (as well as the reverse direction) follows from the previous result. Note that the nontriviality of the solution $u$ in (ii) gives $[u] \neq 0$ by the uniqueness part of Theorem 4.2. The implication (i) $\Longrightarrow$ (iii) follows from Lemma 4.1 . 
A different angle to nontrivial solvability of $(\tilde{L}+1) u=0$ is provided by the geometric context developed in [37] and [6] which we now recall. The setting of [37] and [6] is concerned with locally finite graphs only. However, the part that we need here works in our situation with essentially the same proofs. For the convenience of the reader, we shortly indicate the corresponding proofs. For further discussion we refer to the cited works.

Assume that $(b, c)$ over $(V, m)$ is connected. The length of a path $\gamma=\left(x_{1}, \ldots, x_{n}\right)$ is defined by

$$
L(\gamma) \stackrel{\text { def }}{=} \sum_{j=1}^{n-1} \frac{1}{b\left(x_{j}, x_{j+1}\right)^{1 / 2}} .
$$

Then, $d: V \times V \rightarrow[0, \infty)$ given by

$$
d(x, y)=\inf \{L(\gamma): \gamma \text { is a path connecting } x \text { and } y\}
$$

provides a metric on $V$. Let $\widehat{V}$ be the metric completion of $V$ with respect to $d$ and let $\hat{d}$ be the extension of $d$ to $\widehat{V}$. Note that the completion $\widehat{V}$ may or may not agree with $V$.

The relevance of this metric comes from the fact that

$$
|u(x)-u(y)| \leq Q^{(N)}(u)^{1 / 2} d(x, y)
$$

for any $u \in D\left(Q^{(N)}\right)$ where $Q^{(N)}(u) \stackrel{\text { def }}{=} Q^{(N)}(u, u)$. Indeed, for any path $\gamma=$ $\left(x_{1}, \ldots, x_{n}\right)$ connecting $x$ and $y$, one easily sees by the Cauchy-Schwarz inequality and the subadditivity of the square root that $|u(x)-u(y)| \leq Q^{(N)}(u)^{1 / 2} L(\gamma)$. This gives the desired result.

Equation (2) allows one to extend any $u \in D\left(Q^{(N)}\right)$ to a Lipschitz function $\hat{u}$ on $\widehat{V}$. We define $u_{\infty}$ to be the restriction of $\widehat{u}$ to $V_{\infty} \stackrel{\text { def }}{=} \widehat{V} \backslash V$ if $\widehat{V} \backslash V \neq \emptyset$ and we define $u_{\infty}$ by 0 , otherwise. From the construction and some simple arguments we obtain a continuity property of the map $u \mapsto u_{\infty}$. As this is not included in [6] we discuss it explicitly as follows.

Lemma 4.4. Let $C\left(V_{\infty}\right)$ be the space of continuous functions on the metric space $\widehat{V}$. Then, the map

$$
D\left(Q^{(N)}\right) \longrightarrow C\left(V_{\infty}\right), \quad u \longmapsto u_{\infty},
$$

is continuous when the set on the right hand side is given the topology of locally uniform convergence.

Proof. Let $\left(u_{n}\right)$ be a sequence in $D\left(Q^{(N)}\right)$ converging to $u \in D\left(Q^{(N)}\right)$ with respect to $\langle\cdot, \cdot\rangle_{Q^{(N)}}$. Assume, without loss of generality, that there exists an $o \in V$ with $u_{n}(o)=u(o)$ for all $n \in \mathbb{N}$. Then, from (2) we obtain

$$
\left|\left(u-u_{n}\right)(p)\right|=\left|\left(u-u_{n}\right)(p)-\left(u-u_{n}\right)(o)\right| \leq Q^{(N)}\left(u-u_{n}\right)^{1 / 2} \hat{d}(p, o) .
$$

This gives the desired statement. 
Now, here comes the connection between non-trivial solutions to $(\tilde{L}+1) u=0$ and the boundary values $u_{\infty}$ of the functions $u$ in $Q^{(N)}$. This is our version of Theorem 2.1 of [6].

Theorem 4.5. Let $(V, m)$ be a discrete measure space and $(b, c)$ a graph over $(V, m)$. Let $\mathcal{B}^{(N)}=D\left(Q^{(N)}\right) / D\left(Q^{(D)}\right)$. Then, the map

$$
P: \mathscr{B}^{(N)} \longrightarrow\left\{u_{\infty}: u \in D\left(Q^{(N)}\right)\right\}, \quad[u] \longmapsto u_{\infty},
$$

is well-defined, linear, continuous and onto. In particular, to each $f \in D\left(Q^{(N)}\right)$ there exists $w \in D\left(Q^{(N)}\right)$ with $(\tilde{L}+1) w=0$ and $w_{\infty}=f_{\infty}$. Furthermore, if $\hat{V}$ is compact, then $P$ is injective.

Proof. The second statement on the existence of $w$ follows from the first statement and Theorem 4.2. The last statement follows from a maximum principle as in [6]. Thus, it suffices to show the first statement. It is clear that $P$ is linear and onto (if it is well-defined). Also, from the previous lemma, it is clear that it is continuous (if it is well-defined). Thus, it remains to show that $P$ is well-defined. Obviously, $u_{\infty}=0$ for all $u \in C_{c}(V)$. Thus, by Lemma 4.4, we obtain that $u_{\infty}=0$ for all $u \in D\left(Q^{(D)}\right)$. Hence, $P$ is well-defined.

From this proposition, Corollary 4.3, and Proposition 9.1 we immediately infer the following corollary.

Corollary 4.6. Let $(V, m)$ be a discrete measure space and $(b, c)$ a graph over $(V, m)$. If there exists $f \in D\left(Q^{(N)}\right)$ with $f_{\infty} \neq 0$, then $Q^{(N)} \neq Q^{(D)}$. If, furthermore, (FC) holds, i.e. $\tilde{L} C_{c}(V) \subseteq \ell^{2}(V, m)$, then the restriction of $\widetilde{L}$ to $C_{c}(V)$ is not essentially selfadjoint.

Remarks. (a) The statement on failure of essential selfadjointness in the corollary is a generalization of Theorem 3.1 of [6]. There, the statement is shown for locally finite graphs and $c \equiv 0$. Our proof provides a further piece of information in that it shows that the existence of $f \in D\left(Q^{(N)}\right)$ with $f_{\infty} \neq 0$ implies that $Q^{(N)} \neq Q^{(D)}$.

(b) One may wonder whether $Q^{(N)} \neq Q^{(D)}$ is, in fact, equivalent to existence of $f$ with $f_{\infty} \neq 0$. This, however, is not the case as can be seen by the example in Appendix A. In that situation, we have completeness of the graph (as this completeness does not depend on $m$ ) and $Q^{(N)} \neq Q^{(D)}$.

\section{Characterizing Neumann and Dirichlet Laplacians}

In our setting, it follows from Corollary 3.4, that the Dirichlet Laplacian is the largest operator satisfying $(\mathcal{C})$. This naturally raises the question whether a corresponding characterization can be given for the Neumann Laplacian. In this section, we show 
that this holds true in the case of locally finite graphs. More precisely, we study the set of all Markovian restrictions of $\widetilde{L}$ and show that the Dirichlet Laplacian is the largest one and the Neumann Laplacian is the smallest one (Theorem 5.2). These results (and their proofs) can be seen as analogues to results for the "usual" Laplacians (and diffusion-type operators) on sufficiently smooth subsets of Euclidean space as discussed in Section 3.3 of [15]. As a corollary, we obtain a characterization of the agreement of $Q^{(D)}$ and $Q^{(N)}$ in terms of uniqueness of symmetric Markov processes associated to $\widetilde{L}$ (Corollary 5.6).

We start with a definition.

Definition 5.1. Let $(V, m)$ be a discrete measure space and $(b, c)$ a locally finite graph over $(V, m)$. Then, a non-negative selfadjoint restriction of $\widetilde{L}$ is called Markovian if its associated form is a Dirichlet form. The set of all Markovian restrictions of $\widetilde{L}$ is denoted by $\varepsilon=\varepsilon(b, c, m)$.

Remark. If $(b, c)$ is locally finite, then, by Proposition 3.9, it follows that $L_{M}$ is the adjoint operator of $L_{c}$. Therefore, any selfadjoint $L$ is a restriction of $\widetilde{L}$ if and only if it is an extension of $L_{c}$ and, in this case,

$$
L_{c} \subseteq L \subseteq L_{M}
$$

holds. We can therefore think of restrictions of $\widetilde{L}$ as extensions of $L_{c}$ and this explains our notation $\mathcal{E}$ for a set of restrictions.

Theorem 5.2. Let $(V, m)$ be a discrete measure space and $(b, c)$ a locally finite graph over $(V, m)$. Then,

$$
Q^{(N)} \leq Q \leq Q^{(D)}
$$

holds for any form $Q$ associated to a Markovian restriction $L$ of $\widetilde{L}$.

The proof of this theorem is given after a series of intermediate claims. We will assume that we are given a locally finite graph $(b, c)$ over $(V, m)$ throughout. Moreover, by a slight abuse of notation, we will write

$$
\langle u, v\rangle \stackrel{\text { def }}{=} \sum_{x \in V} u(x) \overline{v(x)} m(x)
$$

for all $u \in C(V)$ and $v \in C_{c}(V)$.

By definition, the form associated to $L \in \mathcal{E}$ is a Dirichlet form. This has the following consequences which will be repeatedly used in the sequel; see [15] and [7] for proofs. For any $\beta>0$, the resolvent

$$
G_{\beta} \stackrel{\text { def }}{=}(L+\beta)^{-1}
$$


is positivity preserving, i.e. maps non-negative functions to non-negative functions. Moreover, for all $1 \leq p \leq \infty$, the map $G_{\beta}$ extends uniquely to a map on $\ell^{p}(V, m)$, again denoted by $G_{\beta}$, with norm not exceeding $\frac{1}{\beta}$ and satisfying

$$
G_{\beta} u=\lim _{n \rightarrow \infty} G_{\beta} u_{n}
$$

whenever $u_{n}, u \geq 0$ with $u_{n} \rightarrow u$ monotonously increasing. The $G_{\beta}$ are obviously selfadjoint on $\ell^{2}(V, m)$ and their extensions have the following symmetry property

$$
\left\langle G_{\beta} u, v\right\rangle=\left\langle u, G_{\beta} v\right\rangle
$$

for any $u \in \ell^{p}(V, m), p \geq 1$, and $v \in C_{c}(V)$.

Proposition 5.3. Let $L \in \mathcal{E}$ and $G_{\beta}=(L+\beta)^{-1}$ for $\beta>0$. For any $u \in \ell^{p}(V, m)$ and $v \in C_{c}(V)$ the equation

$$
\lim _{\beta \rightarrow \infty} \beta\left\langle u-\beta G_{\beta} u, v\right\rangle=\langle\tilde{L} u, v\rangle
$$

holds.

Proof. It suffices to show

$$
\lim _{\beta \rightarrow \infty} \beta\left\langle u-\beta G_{\beta} u, v\right\rangle=\langle u, \widetilde{L} v\rangle .
$$

Then, the claim follows from (1) as $\widetilde{F}=C(V)$ due to local finiteness. We calculate

$$
\begin{aligned}
\beta\left\langle u-\beta G_{\beta} u, v\right\rangle & =\beta\left(\langle u, v\rangle-\beta\left\langle G_{\beta} u, v\right\rangle\right) \\
& \stackrel{(!)}{=} \beta\left\langle G_{\beta} u, L v\right\rangle \\
& =\left\langle u, \beta G_{\beta} L v\right\rangle \stackrel{(! !)}{\longrightarrow}\langle u, L v\rangle .
\end{aligned}
$$

As $L \subseteq L_{M}$ (see above), this shows the desired statement.

Here, (!!) follows from the spectral theorem. The statement (!) follows as, obviously,

$$
\left\langle G_{\beta} u,(L+\beta) v\right\rangle=\langle u, v\rangle
$$

and hence

$$
\left\langle G_{\beta} u, L v\right\rangle=\langle u, v\rangle-\beta\left\langle G_{\beta} u, v\right\rangle .
$$

This finishes the proof.

Proposition 5.4. Let $L \in \mathcal{E}$ and $G_{\beta}=(L+\beta)^{-1}$ for $\beta>0$. Then, for any real-valued $u \in D(Q)$

$$
\beta\left\langle u-\beta G_{\beta} u, u\right\rangle=\frac{1}{2}\left\langle f_{\beta}, 1\right\rangle
$$


where the non-negative function $f_{\beta}: V \rightarrow \mathbb{R}$ belonging to $\ell^{1}(V, m)$ is given by

$$
\begin{aligned}
f_{\beta}(x)= & \beta^{2} G_{\beta}(u(x) 1-u)^{2}(x)+2 \beta u(x)^{2}\left(1-\beta G_{\beta} 1(x)\right) \\
=- & \beta\left(u^{2}(x)-\beta\left(G_{\beta} u^{2}\right)(x)\right)+2 \beta u(x)\left(u(x)-\beta G_{\beta} u(x)\right) \\
& +\beta u^{2}(x)\left(1-\beta G_{\beta} 1(x)\right) .
\end{aligned}
$$

Here, $u(x) 1$ denotes the constant function with value $u(x)$ on $V$.

Proof. We start by discussing the definition of $f_{\beta}$. We first note that both expressions for $f_{\beta}$ make sense as $G_{\beta}$ is applied to (sums of) elements from $\ell^{p}(V, m)$ for $1 \leq p \leq \infty$. Note that the first representation gives that $f_{\beta}$ is non-negative and the second representation gives that $f_{\beta}$ belongs to $\ell^{1}(V, m)$.

Finally, the claimed equalities follow by direct computations. These use

$$
m(x) G_{\beta} w(x)=\left\langle G_{\beta} w, \delta_{x}\right\rangle=\left\langle w, G_{\beta} \delta_{x}\right\rangle
$$

for any $w$ which is a sum of functions in $\ell^{p}(V, m)$ and for $\delta_{x}$, the characteristic function of $\{x\}$.

Proposition 5.5. For any $L \in \mathcal{E}$ with associated form $Q$ the Hilbert space $D(Q)$ with inner product $Q(\cdot, \cdot)+\langle\cdot, \cdot\rangle$ is the orthogonal sum

$$
D(Q)=D\left(Q^{(D)}\right) \oplus \mathscr{H}^{(Q)},
$$

where $\mathscr{H}^{(Q)}=\{u \in D(Q):(\widetilde{L}+1) u=0\}$. In particular, any $u \in D(Q)$ can be decomposed uniquely as $u=u_{0}+h$ with $u_{0} \in D\left(Q^{(D)}\right)$ and $h \in \mathscr{H}^{(Q)}$ and

$$
Q(u, u)+\langle u, u\rangle=Q^{(D)}\left(u_{0}, u_{0}\right)+Q(h, h)+\left\langle u_{0}, u_{0}\right\rangle+\langle h, h\rangle .
$$

Proof. The first statement is an immediate consequence of Theorem 3.6; see [15], Lemma 3.3.2(ii), as well. The second statement is an immediate consequence of the first statement.

After these preparations, we are now ready to give a proof of the main result of this section.

Proof of Theorem 5.2. To avoid tedious but non-essential terms we assume that $c \equiv 0$. The statement on the Dirichlet operator $L^{(D)}$ is clear and has already been discussed in the introduction to this section. We show the statement on the Neumann operator. Thus, let $L \in \mathcal{E}$ be given and $Q$ be the associated Dirichlet form. By Proposition 5.5 (applied to both $Q$ and $Q^{(N)}$ ) it suffices to show that

$$
Q(u, u) \geq Q^{(N)}(u, u)
$$


for all real-valued $u \in D(Q)$ with $(\widetilde{L}+1) u=0$. We will investigate the left hand side and the right hand side of (3) separately. To do so we define $T: V \rightarrow \mathbb{R}$ by

$$
T(x) \stackrel{\text { def }}{=}-\sum_{y \in V} b(x, y)(u(x)-u(y)) u(y) .
$$

Note that $T(x)$ is well-defined as $(b, c)$ is locally finite.

Right hand side of (3). As we do not even know that $u \in D\left(Q^{(N)}\right)$ we have to exercise some care. However, by Fubini's theorem and the local finiteness of $b$, the expression

$$
\begin{aligned}
Q^{(N)}(u, u) & =\frac{1}{2} \sum_{x, y \in V} b(x, y)(u(x)-u(y))(u(x)-u(y)) \\
& =\frac{1}{2} \sum_{x \in V}\left(\sum_{y \in V} b(x, y)(u(x)-u(y)) u(x)\right. \\
& \left.-\sum_{y \in V} b(x, y)(u(x)-u(y)) u(y)\right)
\end{aligned}
$$

is well-defined (i.e. either converges absolutely or diverges to $\infty$ ) and all inner sums converge. Now, using $(\widetilde{L}+1) u=0$ and the absolute convergence of $\sum_{x} u(x)^{2} m(x)$, we obtain

$$
0 \leq Q^{(N)}(u, u)=\frac{1}{2} \sum_{x \in V}\left(-u(x)^{2} m(x)+T(x)\right),
$$

where the sum is well-defined, i.e. either converges absolutely or diverges to $\infty$.

Left hand side of (3). By the spectral theorem and Proposition 5.4 we have that

$$
Q(u, u) \geq \beta\left\langle u-\beta G_{\beta} u, u\right\rangle \geq \frac{1}{2}\left\langle f_{\beta}, v\right\rangle \geq 0
$$

for any $v \in C_{c}(V)$ with $0 \leq v \leq 1$ and any $\beta>0$. Here, we use that $f_{\beta} \geq 0$. Hence, taking $\beta \rightarrow \infty$, by Fatou's lemma, Proposition 5.3 and the second expression for $f_{\beta}$ in Proposition 5.4, we obtain that

$$
Q(u, u) \geq \sum_{x \in V} v(x)\left(-\frac{1}{2}\left(\tilde{L} u^{2}\right)(x)+u \widetilde{L} u(x)\right) m(x) \geq 0 .
$$

Now, a direct computation using $(\widetilde{L}+1) u=0$ shows that

$$
\left(\tilde{L} u^{2}\right)(x)=-u^{2}(x)-\frac{1}{m(x)} T(x)
$$

and

$$
u(x) \tilde{L} u(x)=-u^{2}(x)
$$


Putting this together, we find that

$$
Q(u, u) \geq \frac{1}{2} \sum_{x \in V} v(x)\left(-u^{2}(x) m(x)+T(x)\right) \geq 0 .
$$

As $v \in C_{c}(V)$ with $0 \leq v \leq 1$ was arbitrary, we obtain, in particular, that

$$
\left(-u^{2}(x) m(x)+T(x)\right) \geq 0
$$

for all $x \in V$. This shows that we can take a limit over $v$ with $0 \leq v \leq 1$ and $v \rightarrow 1$ pointwise to obtain

$$
Q(u, u) \geq \frac{1}{2} \sum_{x \in V}\left(-u^{2}(x) m(x)+T(x)\right) .
$$

Comparing this with (4) we obtain that

$$
Q(u, u) \geq Q^{(N)}(u, u)
$$

and the desired statement follows. This finishes the proof.

Remarks. (a) Note that great care has to be exercised when plugging $u$ with $(\tilde{L}+1) u$ $=0$ into $Q^{(N)}$ as, formally,

$$
0 \leq Q^{(N)}(u, u)=\langle\tilde{L} u, u\rangle=-\langle u, u\rangle \leq 0
$$

giving $Q^{(N)}(u, u)=0$ for all such $u$ (which would imply that $u=0$ whenever the graph is connected and $m(V)=\infty$ ).

(b) In general, there will exist Dirichlet forms between $Q^{(D)}$ and $Q^{(N)}$. One way to generate such a form is given as follows. Choose an arbitrary subset $A \subseteq V$. Then, define

$$
D_{A}^{\prime} \stackrel{\text { def }}{=}\left\{u \in D\left(Q^{(N)}\right): \sharp\{x \in A: u(x) \neq 0\}<\infty\right\}
$$

and let $D_{A}$ be the closure of $D_{A}^{\prime}$ in the Hilbert space $\left(D\left(Q^{(N)}\right),\langle\cdot, \cdot\rangle_{Q^{(N)}}\right)$. Then, the restriction of $Q^{(N)}$ to $D_{A}$ will be a Dirichlet form by Theorem 3.1.1 of [15]. In general, it will differ from both $Q^{(N)}$ and $Q^{(D)}$.

(c) The previous remark shows that there exist Dirichlet forms between $Q^{(D)}$ and $Q^{(N)}$. Based on the considerations of this paper one might try and characterize all of these forms (via boundary conditions). We consider this an interesting open problem.

(d) The considerations above use the local finiteness of the graph in various places. It should be interesting to find out whether a similar result holds in the general case as well.

Our considerations give another characterization of $Q^{(D)}=Q^{(N)}$ in the case of locally finite graphs. To state the characterization we introduce one more piece of 
(standard) notation. A map $P$ from $[0, \infty)$ into the set of selfadjoint bounded operators on $\ell^{2}(V, m)$ is called a strongly continuous symmetric semigroup if it has the form $P_{t}=e^{-t L}$ for a selfadjoint $L$ which is bounded below. Here, $e^{-t L}$ is understood in the sense of the spectral calculus for selfadjoint operators. The operator $L$ is called the generator of the semigroup. If the form associated to $L$ is a Dirichlet form, the semigroup is called Markovian.

Corollary 5.6. Let $(V, m)$ be a discrete measure space and $(b, c)$ a locally finite graph over $(V, m)$. Then, the following assertions are equivalent:

(i) $Q^{(D)}=Q^{(N)}$;

(ii) there exists only one strongly continuous symmetric Markovian semigroup with generator contained in $\widetilde{L}$.

Remark. It should be interesting to find out to what extent a similar result may hold for more general Dirichlet forms.

\section{The equation $(\tilde{L}+1) u=0$}

In Section 4 we have seen that the set of solutions of $(\widetilde{L}+1) u=0$ in $D\left(Q^{(N)}\right)$ describes the difference between $Q^{(N)}$ and $Q^{(D)}$. In particular, the disagreement of $Q^{(N)}$ and $Q^{(D)}$ was characterized in terms of nontrivial solvability of $(\widetilde{L}+1) u=0$ in $D\left(Q^{(N)}\right)$. In this section we put these results in perspective by discussing the nontrivial solvability of $(\widetilde{L}+1) u=0$ in the spaces $\ell^{2}(V, m)$ and $\ell^{\infty}(V)$. This turns out to be related to essential selfadjointness and stochastic completeness, respectively. As a consequence, we obtain some immediate connections between the agreement of $Q^{(N)}$ and $Q^{(D)}$, essential selfadjointness and stochastic completeness. By examples, we show that no further implications hold in general.

Before we start the discussion let us note that the number one in the equation $(\widetilde{L}+1) u=0$ does not play any special role. It could be replaced by any positive number $\alpha$. Then, virtually the same arguments apply to solutions of $(\widetilde{L}+\alpha) u=0$. In fact, the arguments apply to any number $\alpha$ with $-\alpha$ smaller than the infimum of the spectrum of $L^{(N)}$. We stick to the case $\alpha=1$ for convenience only.

We now turn to the concept of stochastic completeness. Recall that $(V, b, c, m)$ with $c \equiv 0$ is called stochastically complete if

$$
M_{t} \stackrel{\text { def }}{=} e^{-t L^{(D)}} 1 \equiv 1
$$

for all $t \geq 0$. This can be shown to be equivalent to $(\tilde{L}+1) u=0$ not having a non-trivial solution in $\ell^{\infty}(V)$ (and to various further statements); see [40], [26], and [22]. It turns out that this type of characterization can be extended to the case 
$c \not \equiv 0$ if one is willing to modify $M$. More precisely, in the general case (with not necessarily vanishing $c$ ), one defines, for each $t \geq 0$, the function $M_{t}$ on $V$ by

$$
M_{t} \stackrel{\text { def }}{=} e^{-t L^{(D)}} 1+\int_{0}^{t} e^{-s L^{(D)}} \frac{c}{m} d s .
$$

Here, for the non-negative $c / m$, the function $e^{-t L^{(D)}} c / m$ is defined as a limit by approximating $c / m$ from below by non-negative functions in $C_{c}(V)$; see [26]. The function $M_{t}$ turns out to be finite with values between 0 and 1 . Note that the function agrees with our earlier definition of $M_{t}$ if $c \equiv 0$. We then say that $(V, b, c, m)$ satisfies $\left(S C_{\infty}\right)$ if $M_{t} \equiv 1$ and speak of $\left(S C_{\infty}\right)$ as stochastic completeness at infinity. As shown in [26] and [27], the following holds.

Theorem 6.1 (Characterization of stochastic completeness). Let $(V, m)$ be a discrete measure space and $(b, c)$ a graph over $(V, m)$. Then, the following assertions are equivalent:

(i) $(V, b, c, m)$ is stochastically complete at infinity, i.e. $M_{t} \equiv 1$ for all $t \geq 0$;

(ii) There does not exist a non-trivial solution of $(\tilde{L}+1) u=0$ in $\ell^{\infty}(V)$.

Remark. Note that $M$ is defined using the Dirichlet operator $L^{(D)}$. Analogously, one might define

$$
M_{t}^{(N)} \stackrel{\text { def }}{=} e^{-t L^{(N)}} 1+\int_{0}^{t} e^{-s L^{(N)}} \frac{c}{m} d s .
$$

We then infer that $M \equiv 1 \Rightarrow M^{(N)} \equiv 1$ (as $M \equiv 1$ implies $Q^{(N)}=Q^{(D)}$ by Lemma 4.1 and the previous theorem). However, the reverse implication that $M^{(N)} \equiv 1 \Longrightarrow M \equiv 1$ does not hold. To see this, we can consider the example of Appendix A (see [26] as well) with $c \equiv 0, m(V)<\infty$ and $Q^{(N)} \neq Q^{(D)}$. By $Q^{(N)} \neq Q^{(D)}$, Lemma 4.1 and the preceding theorem, we infer the failure of $M \equiv 1$. On the other hand, by $m(V)<\infty$ we obtain that 1 is eigenfunction of $L^{(N)}$. Thus, $M^{(N)}=e^{-t L^{(N)}} 1 \equiv 1$. This shows that, in terms of processes, stochastic completeness cannot be defined with the "Neumann-process". This seems worth noting as the characterization of stochastic completeness via (un)boundedness of solutions of $(\widetilde{L}+1) u=0$ does not refer to any specific selfadjoint realization of $\tilde{L}$.

We now turn to essential selfadjointness. The following result essentially deals with the deficiency index being zero. In the context of graph Laplacians it could be derived from the considerations of [26]. We include a proof for completeness.

Theorem 6.2 (Characterization of essential selfadjointness). Let $(V, m)$ be a discrete measure space and $(b, c)$ a graphover $(V, m)$. Assume $(\mathrm{FC})$, i.e. $\widetilde{L} C_{c}(V) \subseteq \ell^{2}(V, m)$. Then, the following assertions are equivalent: 
(i) the restriction of $\widetilde{L}$ to $C_{c}(V)$ is essentially selfadjoint;

(ii) there does not exist a non-trivial solution of $(\widetilde{L}+1) u=0$ in $\ell^{2}(V, m)$.

Proof. Recall, from Section 3, the definition of the operator $L_{c}$ as the restriction of $\widetilde{L}$ to $C_{c}(V)$ and the maximal operator $L_{M}$ as the restriction of $\widetilde{L}$ to the set of all $u \in \ell^{2}(V, m)$ with $\widetilde{L} u \in \ell^{2}(V, m)$. Then, by Proposition $3.9, L_{c}$ is a symmetric nonnegative operator with adjoint $L_{M}$. As $L_{c}$ is non-negative, essential selfadjointness is then equivalent to triviality of the kernel of $L_{M}+1$ by standard theory.

We note the following consequence of Lemma 4.1 and the considerations above.

Corollary 6.3. Let $(V, m)$ be a discrete measure space and $(b, c)$ a graph over $(V, m)$.

(a) If the graph is stochastically complete at infinity, then $Q^{(N)}=Q^{(D)}$.

(b) If $\tilde{L}$ maps $C_{c}(V)$ to $\ell^{2}(V, m)$ and $L_{c}$ is essentially selfadjoint, then $Q^{(N)}=$ $Q^{(D)}$.

Abbreviating stochastic completeness at infinity by S.C. and essential selfadjointness by E.S. we can summarize the preceding considerations as follows:

$$
\left.\begin{array}{l}
\text { S.C. } \\
\text { E.S. } \\
Q^{(N)}=Q^{(D)}
\end{array}\right\} \begin{array}{r}
\text { No nontrivial solution } \\
\text { to }(\widetilde{L}+1) u=0 \text { in }
\end{array}\left\{\begin{array}{r}
\ell^{\infty}(V) \\
\ell^{2}(V, m) \text { and }(\mathrm{FC}) \\
D\left(Q^{(N)}\right) \cap \ell^{\infty}(V)
\end{array}\right.
$$

In particular: $E . S . \Longrightarrow Q^{(N)}=Q^{(D)} \Longleftarrow S . C$.

This shows some connections between stochastic completeness, essential selfadjointness and $Q^{(N)}=Q^{(D)}$. It turns out that no further implications hold, i.e. stochastic completeness at infinity and essential selfadjointness are independent. In particular, neither in (a) nor in (b) of Corollary 6.3 does the reverse implication hold. This is now shown by a series of examples.

Example 1 and Example 2 (Graphs satisfying E.S. and S.C. and graphs satisfying E.S. without S.C., respectively). We consider graphs with $m \equiv 1, c \equiv 0$ and $b$ taking values in $\{0,1\}$ only. Then, as shown in [26] and [39], essential selfadjointness holds due to the assumption that $m \equiv 1$.

More specifically, we will now even further restrict attention to radially symmetric rooted trees. Thus, we are given a tree with a root $o$ and all vertices with distance $n$ to the root have the same degree $d_{n}$. Then, as shown in [39], the corresponding models will satisfy S.C. if and only if

$$
\sum_{n} \frac{1}{d_{n}}=\infty .
$$

Thus, within the class of radially symmetric rooted trees, we can easily find examples satisfying E.S. together with S.C. and examples satisfying E.S. without S.C. 
Example 3 (Graphs satisfying neither S.C. nor E. S.). The example in Appendix A (see Section 4 of [26] as well) gives a situation with $m(V)<\infty, c \equiv 0$, and $1 \notin D\left(Q^{(D)}\right)$ implying, in particular, that $Q^{(N)} \neq Q^{(D)}$. Thus, in this example, we have neither essential selfadjointness nor stochastic completeness.

Example 4 (Graphs satisfying S.C. without E.S.). Based on the first example of Section 4 of [26] we may give such a graph as follows. Let $V=\mathbb{Z}$ and $b(x, y)=1$ if $|x-y|=1$ and zero otherwise. The weighted graph $(b, 0)$ over $(V, 1)$ gives rise to the bounded operator $\Delta: \ell^{2}(\mathbb{Z}) \rightarrow \ell^{2}(\mathbb{Z})$ which is the restriction of the formal operator

$$
(\tilde{\Delta} w)(x)=-w(x-1)+2 w(x)-w(x+1)
$$

to $\ell^{2}(\mathbb{Z}) \stackrel{\text { def }}{=} \ell^{2}(\mathbb{Z}, 1)$. As $\Delta$ is bounded (see, e.g., Theorem 9.3 in Section 9 below), it is essentially selfadjoint. Moreover, it is well-known that $(\mathbb{Z}, b, 0,1)$ is stochastically complete (see Examples 1 and 2 and compare, for instance, [12], Theorem 2.10) and, therefore, there is no solution to $(\widetilde{\Delta}+1) w=0$ in $\ell^{\infty}(\mathbb{Z})$.

Let $\lambda \stackrel{\text { def }}{=} \operatorname{arccosh}(3 / 2)$. As $e^{\lambda}+e^{-\lambda}-2=1$, one checks that

$$
u: \mathbb{Z} \longrightarrow[0, \infty), \quad x \longmapsto e^{\lambda x}
$$

is a solution to $(\widetilde{\Delta}+1) u=0$. Choose $\varphi \in \ell^{1}(\mathbb{Z})$ with $\varphi(x)>0$ for all $x \in \mathbb{Z}$. Let the measure $m$ on $\mathbb{Z}$ be given by

$$
m(x)=\min \left\{1, \frac{\varphi(x)}{u^{2}(x)}\right\}
$$

and the $\operatorname{map} c: \mathbb{Z} \rightarrow[0, \infty)$ via

$$
c(x) \stackrel{\text { def }}{=} \max \left\{0, \frac{u^{2}(x)}{\varphi(x)}-1\right\} m(x) .
$$

Then,

$$
1 \equiv c+m
$$

by construction. The graph $(\mathbb{Z}, b, c, m)$ induces the formal operator

$$
(\tilde{L} w)(x)=\frac{1}{m(x)}(-w(x-1)+2 w(x)-w(x+1))+\frac{c(x)}{m(x)} w(x),
$$

or, formally, $\widetilde{L}=\frac{1}{m}(\widetilde{\Delta}+c)$. As $1 \equiv c+m$, one directly checks that $w: \mathbb{Z} \rightarrow \mathbb{R}$ solves

$$
(\tilde{L}+1) w=0 \quad \text { if and only if } \quad(\widetilde{\Delta}+1) w=0 .
$$

We conclude the following. The function $u: x \mapsto e^{\lambda x}$ belongs to $\ell^{2}(\mathbb{Z}, m)$ by the choice of $m$ and $(\widetilde{L}+1) u=0$ since $(\widetilde{\Delta}+1) u=0$. Therefore, the restriction of $\widetilde{L}$ to $C_{c}(\mathbb{Z})$ is not essentially selfadjoint. On the other hand, there is no solution to $(\widetilde{L}+1) w=0$ in $\ell^{\infty}(\mathbb{Z})$ since there is no solution to $(\widetilde{\Delta}+1) w=0$ in $\ell^{\infty}(\mathbb{Z})$ (see above). Hence, $(\mathbb{Z}, b, c, m)$ is stochastically complete. 


\section{Maximum principle and characterization of positivity improvement}

In this section we present a maximum principle and use it to characterize positivity improvement of a positivity preserving semigroup of the form $\left(e^{-t L}\right)_{t \geq 0}$ with $L \subseteq \widetilde{L}$. For the Dirichlet Laplacian this has already been done in [26].

Theorem 7.1 (Maximum principle). Let $(V, m)$ be a discrete measure space, $(b, c)$ a connected graph over $(V, m)$ and $\widetilde{L}=\widetilde{L}_{(b, c, m)}$. Let $w$ be a real-valued solution of $\tilde{L} w \leq 0$. If $w$ attains its maximum and this maximum is non-negative, then $w$ is constant and even $w \equiv 0$ if $c \not \equiv 0$.

Proof. Let $x \in V$ be given such that $w$ attains its non-negative maximum at $x$. From

$$
0 \geq \tilde{L} w(x)=\frac{1}{m(x)} \sum_{y \in V} b(x, y)(w(x)-w(y))+\frac{c(x)}{m(x)} w(x)
$$

we then infer that all $y \in V$ with $b(x, y)>0$ must have $w(x)=w(y)$. Inductively, we obtain the constancy of $w$. Now, a second look at $\widetilde{L} w \leq 0$ shows the last part of the statement.

Corollary 7.2. Let $(V, m)$ and $(b, c)$ be as in the previous theorem. Let $u$ be a non-negative solution to $(\widetilde{L}+1) u \geq 0$. Then, either $u$ is strictly positive or $u \equiv 0$.

Proof. This is a direct consequence of the previous theorem applied with $w=-u$ and $\widetilde{L}$ replaced by $\widetilde{L}+1$.

Recall that a bounded operator $A$ on $\ell^{2}(V, m)$ is positivity preserving if it maps non-negative functions to non-negative functions. It is called positivity improving if it maps non-negative functions which do not vanish identically to strictly positive functions. A semigroup $\left(e^{-t L}\right)_{t \geq 0}$ is said to be positivity preserving and positivity improving, respectively, if, for every $t>0, e^{-t L}$ has the corresponding property.

Theorem 7.3 (Characterization of positivity improvement). Let $(V, m)$ be a discrete measure space and $(b, c)$ a graph over $(V, m)$. Let $L$ be a selfadjoint non-negative restriction of $\tilde{L}$ such that the associated semigroup $\left(e^{-t L}\right)_{t \geq 0}$ is positivity preserving. Then, the semigroup $\left(e^{-t L}\right)_{t \geq 0}$ is positivity improving if and only if $(b, c)$ is connected.

Proof. It is clear that the semigroup cannot be positivity improving if the graph is not connected.

Let us now turn to the other implication. Thus, we assume that the graph $(b, c)$ is connected. By general principles, it suffices to show that the resolvent $(L+1)^{-1}$ is positivity improving. So, let $u \geq 0$ with $u \neq \equiv 0$ be given and consider the function $v \stackrel{\text { def }}{=}(L+1)^{-1} u$. Then, $v$ is non-negative as $e^{-t L}$, and thus $(L+1)^{-1}$, is positivity preserving and satisfies $(\widetilde{L}+1) v=u \geq 0$ as $L$ is a restriction of $\widetilde{L}$. Now, the desired positivity follows from the previous corollary. 


\section{An analogue to a theorem of $\mathrm{Li}$}

Whenever $L$ is a non-negative selfadjoint operator on $\ell^{2}(V, m)$ we can form the associated semigroup $e^{-t L}$. By the discreteness of $V$ these operators have a kernel, i.e. there exists a map

$$
p:[0, \infty) \times V \times V \longrightarrow \mathbb{C}
$$

with

$$
e^{-t L} f(x)=\sum_{y \in V} p_{t}(x, y) f(y) m(y)
$$

for all $f \in \ell^{2}(V, m)$. Thus, with the characteristic function $\delta_{x}$ of $x \in V$ we obtain

$$
\left\langle\delta_{x}, e^{-t L} \delta_{y}\right\rangle=m(x) m(y) p_{t}(x, y)
$$

for all $x, y \in V$. If $L$ arises from a Dirichlet form, then $p$ must be non-negative with $\sum_{y} p_{t}(x, y) m(y) \leq 1$. In this case, estimates of this kernel are of particular interest. Some basic estimates are discussed in the main result of this section. The result is taken from [28], following [2] and [36]. We present an alternative proof of Theorem 8.1(b), which is known as Theorem of $\mathrm{Li}$ in the context of manifolds (after [31]).

Theorem 8.1. Let $(V, m)$ be a discrete measure space and $(b, c)$ a connected graph over $(V, m)$. Let $L$ be a selfadjoint non-negative restriction of $\widetilde{L}$ such that the associated semigroup $\left(e^{-t L}\right)_{t \geq 0}$ is positivity preserving. Furthermore, let $E_{0}$ be the infimum of the spectrum of $L$.

(a) There exists a unique non-negative $\Phi$ on $V$ such that

$$
e^{t E_{0}} p_{t}(x, y) \longrightarrow \Phi(x) \Phi(y), \quad t \rightarrow \infty
$$

for all $x, y \in V$. Here, $\Phi \equiv 0$ if $E_{0}$ is not an eigenvalue and $\Phi$ is the unique normalized positive eigenfunction to $E_{0}$, otherwise.

(b) The kernel $p$ of $e^{-t L}$ satisfies

$$
\frac{\log p_{t}(x, y)}{t} \longrightarrow-E_{0}, \quad t \rightarrow \infty
$$

for all $x, y \in V$.

Proof. Part (a) can be obtained as a simple consequence of the spectral theorem (see [36] and [28]) as follows. Let $P$ be the projection onto the eigenspace of $E_{0}$, i.e. $P=0$ if $E_{0}$ is not an eigenvalue and $P=\langle\Phi, \cdot\rangle \Phi$ otherwise.

Then, the spectral theorem gives

$$
m(x) m(y)\left|e^{t E_{0}} p_{t}(x, y)-\Phi(x) \Phi(y)\right|
$$




$$
\begin{aligned}
& =\left|\left\langle\delta_{x},\left(e^{t E_{0}} e^{-t L}-P\right) \delta_{y}\right\rangle\right| \\
& =\left|\int_{\left[E_{0}, \infty\right)}\left(e^{-t\left(s-E_{0}\right)}-1_{\left\{E_{0}\right\}}(s)\right) d \rho(s)\right| \longrightarrow 0 .
\end{aligned}
$$

Here, $\rho$ is the spectral measure of $L$ associated to $\delta_{x}$ and $\delta_{y}$ characterized by

$$
\left\langle\delta_{x}, f(L) \delta_{y}\right\rangle=\int f(s) d \rho(s)
$$

for all continuous bounded real valued $f$ on $\mathbb{R}$.

(b) Let $\tilde{\delta}_{x}$ with

$$
\tilde{\delta}_{x}(y)=\frac{1}{\sqrt{m(x)}} \delta_{x}(y)
$$

be given, where $\delta_{x}$ is the characteristic function of $\{x\}$. Obviously, $\left\{\tilde{\delta}_{x}\right\}_{x \in V}$ forms an orthonormal basis of $\ell^{2}(V, m)$ consisting of non-negative functions which do not vanish identically. As $(b, c)$ is connected, we infer, from Theorem 7.3, that the semigroup $\left(e^{-t L}\right)_{t \geq 0}$ is positivity improving. Thus, for all $t>0$ and $x, y \in V$, the numbers

$$
a_{t}(x, y) \stackrel{\text { def }}{=}\left\langle\tilde{\delta}_{x}, e^{-t L} \tilde{\delta}_{y}\right\rangle, a_{t}(x) \stackrel{\text { def }}{=} a_{t}(x, x)
$$

are positive and satisfy

$$
a_{t+s}(x)=\left\langle e^{-t L} \tilde{\delta_{x}}, e^{-s L} \tilde{\delta_{x}}\right\rangle=\sum_{y \in V}\left\langle e^{-t L} \tilde{\delta}_{x}, \tilde{\delta}_{y}\right\rangle\left\langle\tilde{\delta}_{y}, e^{-s L} \tilde{\delta}_{x}\right\rangle \geq a_{t}(x) a_{s}(x) .
$$

By a similar reasoning,

$$
a_{t-1}(x) a_{1}(x, y) \leq a_{t}(x, y) \leq \frac{1}{a_{1}(y, x)} a_{t+1}(y)
$$

for all $x, y \in V$ and $t>1$. The first inequality gives the existence of

$$
\lim _{t \rightarrow \infty} \frac{\log a_{t}(x)}{t}
$$

for each $x \in V$ by standard subadditive reasoning. The second line of inequalities then gives that this limit does not depend on $x$ and, in fact,

$$
\lim _{t \rightarrow \infty} \frac{\log a_{t}(x, y)}{t}=E
$$

holds with some real $E$ for all $x, y \in V$. As $\sqrt{m(x) m(y)} p_{t}(x, y)=a_{t}(x, y)$, we obtain the convergence of $\frac{\log p_{t}(x, y)}{t}$ to $E$ for $t \rightarrow \infty$ as well. As this holds for all $x, y \in V$, we obtain $E=-E_{0}$. 
Corollary 8.2. Let $(V, m)$ be a discrete measure space and $(b, c)$ a connected graph over $(V, m)$. Let $L$ be a selfadjoint non-negative restriction of $\widetilde{L}$ such that the associated semigroup $\left(e^{-t L}\right)_{t \geq 0}$ is positivity preserving. Assume that $m(V)=\infty$. Then, the heat kernel associated to L satisfies

$$
p_{t}(x, y) \longrightarrow 0, \quad t \rightarrow \infty .
$$

Proof. Since $m(V)=\infty$ we have that $1 \notin \ell^{2}(V, m)$ and, in particular, that $1 \notin D(Q)$ and 0 is not an eigenvalue. Now, if $E_{0}>0$, then, by the previous theorem, we get that $p_{t}(x, y) \rightarrow 0$ as $t \rightarrow \infty$. Otherwise, if $E_{0}=0$, then the theorem gives that $p_{t}(x, y) \rightarrow \Phi(x) \Phi(y)$ as $t \rightarrow \infty$. But $\Phi \equiv 0$ since 0 is not an eigenvalue.

Remark. Let us emphasize that the assumptions in the preceding results do not entail any form of compact resolvent or spectral gap. Accordingly, one can also not expect any form of exponential rate of convergence in the statements. For situations in which such assumptions and consequences hold we refer to the monograph [13].

\section{Uniqueness of selfadjoint restrictions of $\tilde{L}$}

This section is concerned with two situations in which there is only one selfadjoint restriction of $\widetilde{L}$. This complements the material of the previous sections whose main thrust is the study of situations in which there are several selfadjoint restrictions of $\widetilde{L}$.

We start with the following direct consequence of Proposition 3.9.

Proposition 9.1. Let $(b, c)$ be a graph over the discrete measure space $(V, m)$ and $\widetilde{L}=\widetilde{L}_{(b, c, m)}$. If (FC) holds and the restriction $L_{c}$ of $\widetilde{L}$ to $C_{c}(V)$ is essentially selfadjoint with selfadjoint extension $L$, then $L$ is the only Laplacian associated to $(b, c)$.

Now, we discuss two cases in which the proposition can be applied. One case will involve an assumption on $m$ only and the other case will involve combined assumptions on $m$ and $(b, c)$.

Corollary 9.2. Let $(V, m)$ be a discrete measure space with $\inf _{x \in V} m(x)>0$. Then, (FC) holds for any graph $(b, c)$ over $(V, m)$ and the restriction $L_{c}$ of $\widetilde{L}$ to $C_{c}(V)$ is essentially selfadjoint with selfadjoint extension $L$.

Proof. This follows from the previous proposition by Theorem 6 of [26] and its subsequent remark. Namely, as shown there, $\widetilde{L}$ maps $C_{c}(V)$ to $\ell^{2}(V, m)$ and the restriction of $\widetilde{L}$ to $C_{c}(V)$ is essentially selfadjoint whenever $\inf _{x \in V} m(x)>0$.

Remark. As a consequence of the corollary the main focus of the previous sections concerns the case when $\inf _{x \in V} m(x)=0$. 
Another instance of uniqueness of selfadjoint restrictions of $\widetilde{L}$ is given if $\widetilde{L}$ is a bounded operator. In this context we first discuss an interesting feature of $\widetilde{L}$ as an operator on $\ell^{p}(V, m)$. It is either bounded for all $p \geq 1$ or for no such $p$.

Theorem 9.3. Let $(V, m)$ be a discrete measure space, $(b, c)$ a graph over $(V, m)$ and $\widetilde{L}=\widetilde{L}_{(b, c, m)}$. Then, the following are equivalent:

(i) there exists $a C \geq 0$ with $\frac{1}{m(x)}\left(\sum_{y} b(x, y)+c(x)\right) \leq C$ for all $x \in V$;

(ii) $\widetilde{L}$ gives a bounded operator on $\ell^{p}(V, m)$ for some $p \in[1, \infty]$;

(iii) $\widetilde{L}$ gives a bounded operator on $\ell^{p}(V, m)$ for all $p \in[1, \infty]$.

We begin the proof with an auxiliary claim.

Claim. If $\ell^{p}(V, m)$ is a subset of $\widetilde{F}$ and the restriction of $\tilde{L}$ to $\ell^{p}(V, m)$ is a bounded operator on $\ell^{p}(V, m)$ for some $p$ with $1 \leq p \leq \infty$ and $q$ satisfies $1=1 / p+1 / q$, then $\ell^{q}(V, m)$ also belongs to $\widetilde{F}$ and the restriction of $\widetilde{L}$ to $\ell^{q}(V, m)$ is a bounded operator as well.

Proof of the claim. This is essentially a consequence of (1) and duality. We consider the cases $p=1$ and $p>1$ separately.

To treat the case $p=1$ we note that $\ell^{\infty}(V)$ belongs to $\widetilde{F}$ anyway. From (1) we then infer for $u \in \ell^{\infty}(V)$ and $v \in C_{c}(V)$ the estimate

$$
\left|\sum_{x}(\tilde{L} u)(x) v(x) m(x)\right|=\left|\sum_{x} u(x) \tilde{L} v(x) m(x)\right| \leq C\|u\|_{\infty}\|v\|_{1},
$$

where $\|\cdot\|_{s}$ denotes the $s$-norm on $\ell^{s}(V, m)$ and $C$ is a bound for the norm of $\tilde{L}$ as an operator from $\ell^{1}(V, m)$ to $\ell^{1}(V, m)$. As $C_{c}(V)$ is dense in $\ell^{1}(V, m)$ we infer that $\widetilde{L}$ is a bounded operator on $\ell^{\infty}(V)$ (with norm bounded by $C$ as well).

To treat the case $p>1$ we chose $u \in C_{c}(V) \subset \ell^{q}(V, m)$ and $v \in \ell^{p}(V, m)$. Then, (1) gives the estimate

$$
\left|\sum_{x}(\tilde{L} u)(x) v(x) m(x)\right|=\left|\sum_{x} u(x) \tilde{L} v(x) m(x)\right| \leq C\|u\|_{q}\|v\|_{p},
$$

where $C$ is a bound for the norm of $\widetilde{L}$ as an operator from $\ell^{p}(V, m)$ to $\ell^{p}(V, m)$. This shows that $\widetilde{L} u$ belongs to $\ell^{q}(V, m)$ and satisfies $\|\widetilde{L} u\|_{q} \leq C\|u\|_{q}$. Hence, the restriction of $\tilde{L}$ to $C_{c}(V)$ is a bounded operator (with respect to $\|\cdot\|_{q}$ ). It can then be (uniquely) extended to a bounded operator on $\ell^{q}(V, m)$ and this extension can easily be seen to be a restriction of $\tilde{L}$. This finishes the proof of the claim.

Let us now turn to the actual proof. 
Proof of Theorem 9.3. Assume that (i) is satisfied. Then, we see that $\widetilde{L}$ is a bounded operator on $\ell^{\infty}$ which gives (ii). The auxiliary claim now gives that $\widetilde{L}$ is bounded on $\ell^{1}$. Applying the Riesz-Thorin interpolation theorem, we get (iii).

Assume, conversely, that (ii) is fulfilled. Again by the auxiliary claim, $\tilde{L}$ is also a bounded operator on $\ell^{q}$, where $1=\frac{1}{p}+\frac{1}{q}$. As 2 must belong to the interval between $p$ and $q$ we can use interpolation once more, to get that $\tilde{L}$ is bounded on $\ell^{2}$. Hence, for each $x \in V$, we have the existence of $C \geqslant 0$ such that

$$
\left\langle\tilde{L} \delta_{x}, \delta_{x}\right\rangle \leq C m(x),
$$

which gives (i).

Remark. The equivalence of (i) and boundedness of $\tilde{L}$ on $\ell^{2}(V, m)$ has been shown in [27]. There it has also been shown that this implies boundedness of $\widetilde{L}$ on all $\ell^{p}(V, m)$.

Corollary 9.4. Let $(V, m)$ be a discrete measure space, $(b, c)$ a graph over $(V, m)$ and $\widetilde{L}=\widetilde{L}_{(b, c, m)}$. If there exists $a C \geq 0$ with

$$
\frac{1}{m(x)}\left(\sum_{y \in V} b(x, y)+c(x)\right) \leq C
$$

for all $x \in V$, then there exists only one selfadjoint Laplacian associated to $(b, c)$. This Laplacian is the restriction of $\widetilde{L}$ to $\ell^{2}(V, m)$.

Proof. By definition, any Laplacian associated to the graph is a restriction of $\widetilde{L}$. As the restriction of $\widetilde{L}$ to $\ell^{2}(V, m)$ is bounded by the assumption and the previous theorem, the statement follows.

Remark. Let us point out that the corollary gives examples with uniqueness of the selfadjoint operator associated to $\widetilde{L}$ even if $\inf _{x \in V} m(x)=0$.

\section{Appendix A. A (counter)example}

In this section we briefly recall an example from Section 4 of [26]. This serves as a (counter)example in various situations discussed in the article.

We consider connected graphs $(b, c)$ over $(V, m)$ with $c \equiv 0$ and $b(x, y) \in\{0,1\}$ for all $x, y \in V$. The Cheeger constant $\alpha=\alpha(V, b, c)$ of such a graph is defined by

$$
\alpha \stackrel{\text { def }}{=} \inf _{K \subseteq V: \sharp K<\infty} \frac{Q^{(D)}\left(1_{K}, 1_{K}\right)}{\sharp K},
$$

where $\sharp K$ denotes the cardinality of $K$. The degree $D: V \longrightarrow[0, \infty)$ is defined by $D(x)=\sum_{y \in V} b(x, y)$. Then, as discussed by Dodziuk-Kendall [11] (see [10], 
[25], and [27] as well) in the context of isoperimetric inequalities, the inequality

$$
\frac{1}{2} \sum_{x, y \in V} b(x, y)(\varphi(x)-\varphi(y))^{2} \geq \frac{\alpha^{2}}{2} \sum_{x \in V} D(x) \varphi(x)^{2}
$$

holds for all real-valued $\varphi \in C_{c}(V)$. Now, take an arbitrary graph with $\alpha>0$ and $D(x) \leq C$ for all $x \in V$. To be specific, one may take the regular tree with degree $k \geq 3$. Choose a measure $m$ on $V$ with $m(V)=1$. Then, the graph $(V, b, 0, m)$ has the following features.

(1) The graph $(V, b, 0, m)$ is complete with respect to the metric $d$ defined in Section 4.

(2) The constant function 1 belongs to $D\left(Q^{(N)}\right)$ but not to $D\left(Q^{(D)}\right)$.

Here, claim (1) is clear as $D$ is uniformly bounded. Claim (2) is shown in [26]. We include a short proof. Let 1 be the constant function with value one. As $m(V)<\infty$, the function 1 belongs to $\ell^{2}(V, m)$. Obviously, $Q^{(N)}(1) \stackrel{\text { def }}{=} Q^{(N)}(1,1)=0<\infty$. Thus, $1 \in D\left(Q^{(N)}\right)$. Now, fix $x_{0} \in V$ and let $\varphi_{n}$ be any sequence in $C_{c}(V)$ converging to 1 in $\ell^{2}(V, m)$. Then, $\varphi_{n}\left(x_{0}\right)$ converges to 1 . In particular,

$$
Q^{(N)}\left(\varphi_{n}\right) \geq \frac{\alpha^{2}}{2} D\left(x_{0}\right) \varphi_{n}\left(x_{0}\right)^{2} \longrightarrow \frac{\alpha^{2}}{2} D\left(x_{0}\right)>0, \quad n \rightarrow \infty .
$$

Thus, $Q^{(N)}\left(\varphi_{n}\right)$ does not converge to $0=Q^{(N)}(1)$ and 1 does not belong to $D\left(Q^{(D)}\right)$.

\section{Appendix B. Dirichlet forms on real and complex Hilbert spaces}

In this appendix we shortly discuss some basic and very well-known characterizations of Dirichlet forms. Such forms can be considered on both real and complex Hilbert spaces. Here, we show, in particular, how to go from one situation to the other. We refer to [1], [15], and [34] for further details.

Let $(X, m)$ be a $\sigma$-finite measure space. Let $L^{2}(X, m)$ be the Hilbert space of square integrable function on $X$ with inner product

$$
\langle f, g\rangle=\int_{X} f(x) \overline{g(x)} d m(x) .
$$

As discussed in the main text, a non-negative closed symmetric form $Q$ on $L^{2}(X, m)$ with domain $D(Q)$ is called a Dirichlet form $\left(\right.$ on $\left.L^{2}(X, m)\right)$ if

$$
Q(C u, C u) \leq Q(u, u)
$$

for all $u \in L^{2}(X, m)$ and every normal contraction $C$ on $\mathbb{C}$. Here, we set $Q(v, v)=$ $\infty$ if $v$ does not belong to the form domain. 
In order to state the next theorem, we need some further notation. For a real valued $u$ we set

$$
u_{+} \stackrel{\text { def }}{=} \max \{0, u\} \text { and } u \wedge 1 \stackrel{\text { def }}{=} \min \{1, u\} .
$$

Then, the following theorem holds.

Theorem B.5. Let $Q$ be a non-negative closed symmetric form on $L^{2}(X, m)$. Then, the following assertions are equivalent:

(i) $Q$ is a Dirichlet form;

(ii) the semigroup $e^{-t L}$ is positivity preserving and contracting on $L^{p}(X, m)$ for all $1 \leq p \leq \infty$

(iii) $D(Q)$ is invariant under complex conjugation with $Q(u, v)$ real valued for all real valued $u, v$ in the domain of $Q$ and it holds that $Q\left(u_{+}, u_{+}\right) \leq Q(u, u)$ for all real valued $u \in L^{2}(X, m)$ as well as $Q(u \wedge 1, u \wedge 1) \leq Q(u, u)$ for all real valued $u \in L^{2}(X, m)$ with $u \geq 0$.

Proof. The equivalence of (i) and (ii) follows directly from Theorem XIII.50 and Theorem XIII.51 in Appendix 1 to Section XIII.12 of [35].

Also, it is not hard to see that $D(Q)$ is invariant under complex conjugation with $Q(u, v)$ real valued for all real valued $u, v \in D(Q)$ if and only if $e^{-t L}$ maps real valued functions to real valued functions. Given this, the equivalence between (ii) and (iii) follows again directly from Theorems XIII.50 and XIII.51 in Appendix 1 to Section XIII.12 of [35].

Let $L_{\mathbb{R}}^{2}(X, m)$ be the subspace of $L^{2}(X, m)$ consisting of real-valued functions. We then call a non-negative closed symmetric form $Q$ on $L_{\mathbb{R}}^{2}(X, m)$ a Dirichlet form (on $\left.L_{\mathbb{R}}^{2}(X, m)\right)$ if

$$
Q(C u, C u) \leq Q(u, u)
$$

for all $u \in L_{\mathbb{R}}^{2}(X, m)$ and every normal contraction $C$ on $\mathbb{R}$. (Here, we again set $Q(v, v)=\infty$ if $v$ does not belong to the form domain.) Essentially from (iii) of the previous theorem we obtain the following corollary.

Corollary B.6. (a) Let $Q$ be a Dirichlet form on $L^{2}(X, m)$. Then, the restriction of $Q$ to $L_{\mathbb{R}}^{2}(X, m)$ is a Dirichlet form as well.

(b) Let $Q_{r}$ be a Dirichlet form on $L_{\mathbb{R}}^{2}(X, m)$. Then, the form $Q$ with domain

$$
D(Q) \stackrel{\text { def }}{=}\left\{u+i v: u, v \in D\left(Q_{r}\right)\right\}
$$

and

$$
Q\left(u_{1}+i v_{1}, u_{2}+i v_{2}\right) \stackrel{\text { def }}{=} Q_{r}\left(u_{1}, u_{2}\right)+Q_{r}\left(v_{1}, v_{2}\right)+i\left(Q_{r}\left(v_{1}, u_{2}\right)-Q_{r}\left(u_{1}, v_{2}\right)\right)
$$

is a Dirichlet form on $L^{2}(X, m)$. 
Proof. (a) By (iii) of the previous theorem, $Q(u, v)$ is real valued for all real valued $u, v \in D(Q)$. Now, (i) of the previous theorem, shows that the restriction of $Q$ to real valued functions is compatible with taking normal contractions on $\mathbb{R}$.

(b) It is not hard to see that $Q$ is a symmetric closed non-negative form. Moreover, as $Q_{r}$ is compatible with contractions on $\mathbb{R}$ it is easy to see that (iii) of the previous theorem holds for $Q$. Thus, the previous theorem shows that $Q$ is a Dirichlet form.

\section{References}

[1] N. Bouleau and F. Hirsch, Dirichlet forms and analysis on Wiener space. De Gruyter Studies in Mathematics 14. Walter de Gruyter \& Co., Berlin, 1991. MR 1133391 Zbl 0748.60046

[2] I. Chavel and L. Karp, Large time behavior of the heat kernel: the parabolic $\lambda$-potential alternative. Comment. Math. Helv. 66 (1991), 541-556. MR 1129796 Zbl 0754.58039

[3] F. R. K. Chung, Spectral graph theory. CBMS Regional Conference Series in Mathematics 92. Amer. Math. Soc., Providence (RI), 1997. MR 1421568 Zbl 0867.05046

[4] F. Chung, A. Grigor'yan, and S.-T. Yau, Higher eigenvalues and isoperimetric inequalities on Riemannian manifolds and graphs. Comm. Anal. Geom. 8 (2000), 969-1026. MR 1846124 Zbl 1001.58022

[5] Y. Colin de Verdière, Spectres de graphes. Cours Spécialisés 4. Société Mathématique de France, Paris, 1998. MR 1652692 Zbl 0913.05071

[6] Y. Colin de Verdière, N. Torki-Hamza, and F. Truc, Essential self-adjointness for combinatorial Schrödinger operators II - Metrically non complete graphs. Math. Phys. Anal. Geom. 14 (2011), 21-38. MR 2782792 Zbl 05987646

[7] E. B. Davies, Heat kernels and spectral theory. Cambridge Tracts in Mathematics 92. Cambridge University Press, Cambridge, 1990. MR 1103113 Zbl 0699.35006

[8] E. B. Davies, Linear operators and their spectra. Cambridge Studies in Advanced Mathematics 106. Cambridge University Press, Cambridge, 2007. MR 2359869 Zbl 1138.47001

[9] J. Dodziuk, Difference equations, isoperimetric inequality and transience of certain random walks. Trans. Amer. Math. Soc. 284 (1984), 787-794. MR 0743744 Zbl 0512.39001

[10] J. Dodziuk, Elliptic operators on infinite graphs. In B. Booß-Bavnbek et al. (eds.), Analysis, geometry and topology of elliptic operators. Papers of a workshop in honor of Krzysztof P. Wojciechowski on his 50 ${ }^{\text {th }}$ birthday, Roskilde, Denmark, May 20-22, 2005. World Scientific, Hackensack (NJ), 2006, 353-368. MR 2246774 Zbl 1127.58034

[11] J. Dodziuk and W. S. Kendall, Combinatorial Laplacians and isoperimetric inequality. In K. D. Elworthy, From local times to global geometry, control and physics (Coventry, 1984/85). Pitman Res. Notes Math. Ser. 150. Longman Sci. Tech., Harlow, 1986, pp. 68-74. MR 0894523 Zbl 0619.05005 
[12] J. Dodziuk and V. Mathai, Kato's inequality and asymptotic spectral properties for discrete magnetic Laplacians. In J. Jorgenson and L. Walling (eds.), The ubiquitous heat kernel. Papers from a special session of the AMS Meeting held in Boulder, CO, October 2-4, 2003. Contemporary Mathematics 398. Amer. Math. Soc., Providence (RI), 2006, pp. 69-81. MR 2218014 Zbl 1207.81024

[13] K.-J. Engel and R. Nagel, A short course on operator semigroups. Universitext. Springer, New York, 2006. MR 2229872 Zbl 1106.47001

[14] R. L. Frank, D. Lenz, and D. Wingert, Intrinsic metrics for non-local symmetric Dirichlet forms and applications to spectral theory. Preprint 2010. arXiv:1012.5050

[15] M. Fukushima, Y. Ōshima, and M. Takeda, Dirichlet forms and symmetric Markov processes. De Gruyter Studies in Mathematics 19. Walter de Gruyter \& Co., Berlin, 1994. MR 1303354 Zbl 0838.31001

[16] K. Fujiwara, The Laplacian on rapidly branching trees. Duke Math. J. 83 (1996), 191-202. MR 1388848 Zbl 0856.58044

[17] S. Golénia, Unboundedness of adjacency matrices of locally finite graphs. Lett. Math. Phys. 93 (2010), 127-140. MR 2679965 Zbl 05788007

[18] S. Golénia and C. Schumacher, The problem of deficiency indices for discrete Schrödinger operators on locally finite graphs. J. Math. Phys. 52 (2011). Article id. 063512. MR 2841768

[19] A. Grigor'yan, X. Huang, and J. Masamune, On stochastic completeness of jump processes. To appear in Math. Z. Preprint 2011. www.math.uni-bielefeld.de//grigor/vgg.pdf

[20] S. Haeseler, Heat kernel estimates and related inequalities on metric graphs. Preprint 2010. arXiv:1101.3010

[21] S. Haeseler and M. Keller, Generalized solutions and spectrum for Dirichlet forms on graphs. In [30], 181-200. Zbl 1227.47023

[22] X. Huang, Stochastic incompleteness for graphs and weak Omori-Yau maximum principle. J. Math. Anal. Appl. 379 (2011), 764-782. MR 2784357 Zbl 1229.60088

[23] P. E. T. Jorgensen, Essential self-adjointness of the graph-Laplacian. J. Math. Phys. 49 (2008). Article id. 073510. MR 2432048 Zbl 1152.81496

[24] P. E. T. Jorgensen and E. P. J. Pearse, Spectral reciprocity and matrix representations of unbounded operators. J. Funct. Anal. 261 (2011), 749-776. MR 2799579 Zbl 05907562

[25] M. Keller, The essential spectrum of the Laplacian on rapidly branching tessellations. Math. Ann. 346 (2010), 51-66. MR 2558886 Zbl 05661733

[26] M. Keller and D. Lenz, Dirichlet forms and stochastic completeness of graphs and subgraphs. To appear in J. Reine Angew. Math. Preprint 2011. arXiv:0904.2985

[27] M. Keller and D. Lenz, Unbounded Laplacians on graphs: basic spectral properties and the heat equation. Math. Model. Nat. Phenom. 5 (2010), 198-224. MR 2662456 Zbl 1207.47032

[28] M. Keller, D. Lenz, H. Vogt, and R. Wojciechowski, Note on basic features of large time behaviour of heat kernels. Preprint 2011. arXiv:101.0373

[29] M. Keller and N. Peyerimhoff, Cheeger constants, growth and spectrum of locally tessellating planar graphs. Math. Z. 268 (2011), 871-886. MR 2818734 Zbl 05944193 
[30] D. Lenz, F. Sobieczky, and W. Woess (eds.), Random walks, boundaries and spectra. Proceedings of the workshop on boundaries, Graz, Austria, June 29-July 3, 2009 and the Alp-workshop, Sankt Kathrein, Austria, July 4-5, 2009. Progress in Probability 64. Birkhäuser, Basel, 2011. Zbl 1214.05001

[31] P. Li, Large time behavior of the heat equation on complete manifolds with nonnegative Ricci curvature. Ann. of Math. (2) 124 (1986), 1-21. MR 0847950 Zbl 0613.58032

[32] J. Masamune, A Liouville property and its application to the Laplacian of an infinite graph. In M. Kotani, H. Naito, and T. Tate (eds.), Spectral analysis in geometry and number theory. Papers from the International Conference on the occasion of Toshikazu Sunada's $60^{\text {th }}$ birthday held at Nagoya University, Nagoya, August 6-10, 2007. Contemporary Mathematics 484. Amer. Math. Soc., Providence (RI), 2009, pp. 103-115. MR 1500141 Zbl 1181.58018

[33] B. Mohar, Isoperimetric inequalities, growth, and the spectrum of graphs. Linear Algebra Appl. 103 (1988), 119-131. MR 0943998 Zbl 0658.05055

[34] E. M. Ouhabaz, Analysis of heat equations on domains. London Mathematical Society Monographs Series 31. Princeton University Press, Princeton (NJ), 2005. MR 2124040 Zbl 1082.35003

[35] M. Reed and B. Simon, Methods of modern mathematical physics IV. Analysis of operators. Academic Press, New York etc., 1978. MR 0493420 Zbl 0308.47002

[36] B. Simon, Large time behavior of the heat kernel: on a theorem of Chavel and Karp. Proc. Amer. Math. Soc. 118 (1993), 513-514. MR 1139473 Zbl 0789.58075

[37] N. Torki-Hamza, Laplaciens de graphes infinis I-Graphes métriquement complets. Confluentes Math. 2 (2010), 333-350. Zbl 1203.05109 MR 2740044

[38] A. Weber, Analysis of the physical Laplacian and the heat flow on a locally finite graph. J. Math. Anal. Appl. 370 (2010), 146-158. MR 1214.35075 Zbl 1214.35075

[39] R. K. Wojciechowski, Stochastic completeness of graphs. ProQuest LLC. Ph.D. Thesis. City University of New York, New York, 2008. MR 2711706

[40] R. K. Wojciechowski, Heat kernel and essential spectrum of infinite graphs. Indiana Univ. Math. J. 58 (2009), 1419-1441. MR 2542093 Zbl 1231.05186

[41] R. K. Wojciechowski, Stochastically incomplete manifolds and graphs. In [30], 163-180. Zbl 1221.39008

Received October 16, 2011; revised March 25, 2012

Sebastian Haeseler, Mathematisches Institut, Friedrich Schiller Universität Jena, 07743 Jena, Germany

E-mail: sebastian.haeseler@uni-jena.de

Matthias Keller, Mathematisches Institut, Friedrich Schiller Universität Jena, 07743 Jena, Germany

E-mail: m.keller@uni-jena.de

Daniel Lenz, Mathematisches Institut, Friedrich Schiller Universität Jena, 07743 Jena, Germany. URL: www.analysis-lenz.uni-jena.de/

E-mail: daniel.lenz@uni-jena.de 
Radosław Wojciechowski, Department of Mathematics and Computer Science, York College of The City University of New York, Jamaica, NY 11451, U.S.A.

E-mail: rwojciechowski@gc.cuny.edu 